\title{
Ambit processes; with applications to turbulence and tumour growth
}

\author{
Ole E. Barndorff-Nielsen and Jürgen Schmiegel \\ Thiele Centre for Applied Mathematics in Natural Science, Department of \\ Mathematical Sciences, University of Aarhus, DK-8000 Aarhus, Denmark
}

Summary. The concept of ambit processes is outlined. Such stochastic processes are of interest in spatio-temporal modelling, and they play a central role in recent studies of velocity fields in turbulence and of the growth of cancer tumours. These studies are reviewed, and some open problems are outlined.

Keywords: Brownian sheet; Lévy basis; normal inverse Gaussian; quadratic variation; refined similarity hypotheses; spatio-temporal modelling; stochastic differentials; stochastic integration; stochastic intermittency; tumour growth; turbulence.

\section{Introduction}

The concept of ambit processes discussed in this paper arose out of a current study (Barndorff-Nielsen and Schmiegel (2005), Schmiegel et al (2006), Barndorff-Nielsen and Schmiegel (2004), Schmiegel et al (2004) and Schmiegel (2005a)) the ultimate aim of which is to build a realistic stochastic process model of 3-dimensional turbulent velocity fields, in the spirit of Kolmogorov's phenomenological theory (Frisch (1995)) - and beyond. Besides applications to turbulence, the concept has also been used in modelling the growth of cancer tumours (Schmiegel (2005b)), and it should be of interest to other fields as well.

Section 2 outlines the idea of ambit processes and lists a number of basic questions that need to be resolved in order to have a fullfledged stochastic analysis theory for such processes. In some important special settings, relevant for the turbulence context, the questions can be answered positively. Section 3 provides some background on the physics of turbulence while Section 4 discusses the phenomenology of turbulence. We then, in Section 5, turn to the formulation of a stochastic modelling framework for the velocity and intermittency fields, using the idea of ambit sets, and we outline how it is 
possible within this framework to capture main features of the phenomenological theory. Applications to cancer growth are briefly indicated in Section 6. Section 7 concludes.

\section{Ambit processes}

In this Section we consider a rather general type of spatio-temporal processes that we shall refer to as ambit processes. We do not, at present, have a strict mathematical specification of what should be called an ambit process, but processes of the kind we have in mind would seem to be of interest in a variety of situations, and have in fact been applied not only in turbulence (cf. Section 5) but also for modelling cancer growth (see Section 6).

\subsection{On spatio-temporal processes}

Let $t$ denote time and $\sigma$ a point in some space $\mathcal{S}$. To each point $(t, \sigma) \in \mathbb{R} \times \mathcal{S}$ let there be associated a random variable $Y_{t}(\sigma)$. Let $\omega(w)=(t(w), \sigma(w))$, where $-\infty<w<\infty$, be a smooth curve in space-time, such that $w \rightarrow t(w)$ is nondecreasing, and let $X_{w}=Y_{t(w)}(\sigma(w))$. We assume that $X=\left\{X_{w}\right\}_{w \in \mathbb{R}}$ is welldefined as a stochastic process. Unless otherwise specified we let $\mathcal{S}=\mathbb{R}$.

A key question is when the quadratic variation $[X]$ of $X$ is well-defined in the sense of being a stochastic process such that

$$
[X]_{w}=\mathrm{p}-\lim \sum\left(X_{w_{j}}-X_{w_{j-1}}\right)^{2}
$$

for any sequence of subdivisions $0=w_{0}<w_{1}<\cdots<w_{j}=w$ with $\max \left(w_{j}-w_{j-1}\right) \rightarrow 0$. Intimately connected to this is the question of whether it is possible to define stochastic differentials $\mathrm{d} X_{w}$ and an associated symbolic calculus under which $\left(\mathrm{d} X_{w}\right)^{2}=\mathrm{d}[X]_{w}$.

In settings such that $X_{w}=Y_{t(w)}(\sigma(w))$ is a semimartingale or a linear combination of semimartingales the existence of $[X]$ and of such differentials is of course ensured.

\subsection{Ambit sets and Lévy bases}

Turning now to a more specific setting, suppose that to each point $(t, \sigma)$ is associated a set $A_{t}(\sigma)$, which we refer to as an ambit set. We take $A_{t}(\sigma)$ to be of the form

$$
A_{t}(\sigma)=\left\{(s, \rho): s \leq t, \sigma-c_{t}^{-}(s ; \sigma) \leq \rho \leq \sigma+c_{t}^{+}(s ; \sigma)\right\}
$$

for some nonnegative functions $c_{t}^{-}(s ; \sigma)$ and $c_{t}^{+}(s ; \sigma)$.

A particularly simple case is that of a homogeneous family of ambit sets where 
Ambit processes; with applications to turbulence and tumour growth

$$
A_{t}(\sigma)=\left\{(s, \rho):(s-t, \rho-\sigma) \in A_{0}(0)\right\}
$$

in which case $c_{t}^{-}(s ; \sigma)$ and $c_{t}^{+}(s ; \sigma)$ are independent of $\sigma$ and of the form

$$
c_{t}^{ \pm}(s ; \sigma)=c_{t-s}^{ \pm} .
$$

We write the cumulant function of an arbitrary random variable $X$ as

$$
\mathrm{C}\{\zeta \ddagger X\}=\log \mathrm{E}\left\{e^{i \zeta X}\right\}
$$

and denote the $m$-th order cumulant of $X$ by $c_{m}(X)$, i.e.

$$
c_{m}(X)=i^{m} \frac{\mathrm{d}^{m} \mathrm{C}\{\zeta \ddagger X\}}{\mathrm{d} \zeta^{m}} .
$$

Let $L$ be a Lévy basis, i.e. an independently scattered random measure whose values are infinitely divisible. Then $L$ has a Lévy-Khintchine representation

$$
\mathrm{C}\{\zeta \ddagger L(B)\}=i \zeta a(B)-\frac{1}{2} \zeta^{2} b(B)+\int_{\mathbb{R}}\left\{e^{i \zeta x}-1-i \zeta x \mathbf{1}_{[-1,1]}(x)\right\} \mu(\mathrm{d} x, B)
$$

where $a$ is a signed measure, $b$ is a measure, and $\mu(\mathrm{d} x, B)$ is (for fixed $B$ ) a Lévy measure on $\mathbb{R}$ and a measure for fixed $\mathrm{d} x$. Heuristically it is useful to express (7) in infinitesimal form as

$$
\mathrm{C}\{\zeta \ddagger L(\mathrm{~d} z)\}=i a(\mathrm{~d} z)-\frac{1}{2} \zeta^{2} b(\mathrm{~d} z)+\int_{\mathbb{R}}\left\{e^{i \zeta x}-1-i \zeta x \mathbf{1}_{[-1,1]}(x)\right\} \mu(\mathrm{d} x ; \mathrm{d} z) .
$$

If the Lévy basis $L$ is such that $L(B)$ is Poisson distributed for all $B$ then $L$ is a Poisson basis. In this case the generalised Lévy measure is of the form $\mu(\mathrm{d} x, B)=\operatorname{Leb}(B) \delta_{1}(\mathrm{~d} x)$ where Leb denotes Lebesgue measure and $\delta_{1}$ is the Dirac measure at 1 .

The Lévy basis is said to be factorisable provided $\mu$ factorises as

$$
\mu(\mathrm{d} x, \mathrm{~d} z)=\nu(\mathrm{d} x) c(\mathrm{~d} z)
$$

for some $\sigma$-finite measure $c(\mathrm{~d} z)$ and where $\nu$ is a Lévy measure on $\mathbb{R}$. If, moreover, $a, b$ and $c$ are proportional to Lebesgue measure then $L$ is called homogeneous.

The Brownian sheet is the homogeneous Lévy basis on $\mathbb{R}^{2}$ with $a(\mathrm{~d} z)=0$, $b(\mathrm{~d} z)=\operatorname{Leb}(\mathrm{d} z)$ and $\mu(\mathrm{d} x ; \mathrm{d} z)=0$ in $(8)$.

Remark 1. Integration of deterministic functions with respect to Lévy bases is discussed in detail in Rajput and Rosinski (1989). Here we shall need more general types of integration. However, for the time being we shall argue under the presumption that all the integrals and differentials, and the manipulations with these, are rigourously justifiable, taking up the questions of rigour briefly in Section 2.5. 


\subsection{Ambit processes}

Let $\left\{Y_{t}(\sigma)\right\}_{t \in \mathbb{R}}$ be a spatio-temporal stochastic process of the form

$$
\begin{aligned}
Y_{t}(\sigma)=\mu+\int_{A_{t}(\sigma)} g(t-s, \rho-\sigma) & I_{s}(\rho) L(\mathrm{~d} s \mathrm{~d} \rho) \\
& +\int_{D_{t}(\sigma)} h(t-s, \rho-\sigma) J_{s}(\rho) \mathrm{d} s \mathrm{~d} \rho
\end{aligned}
$$

where $\mu$ is a constant, $\left\{A_{t}(\sigma):(t, \sigma) \in \mathbb{R}^{2}\right\}$ and $\left\{D_{t}(\sigma):(t, \sigma) \in \mathbb{R}^{2}\right\}$ are families of ambit sets, $g$ and $h$ are damping functions (ensuring the convergence of the integrals), $I_{s}(\sigma)$ and $J_{s}(\sigma)$ are random fields on $\mathbb{R}^{2}$, and $L$ is a Lévy basis on $\mathbb{R}^{2}$. A related type of process $\left\{Y_{t}(\sigma)\right\}_{t \geq 0}$ defines

$$
\begin{aligned}
Y_{t}(\sigma)=Y_{0}(\sigma)+\int_{A_{t}^{+}(\sigma)} g(t-s, \rho-\sigma) I_{s}(\rho) L(\mathrm{~d} s \mathrm{~d} \rho) \\
+\int_{D_{t}^{+}(\sigma)} h(t-s, \rho-\sigma) J_{s}(\rho) \mathrm{d} s \mathrm{~d} \rho
\end{aligned}
$$

where $A_{t}^{+}(\sigma)=\left\{(s, \rho) \in A_{t}(\sigma): t \geq 0\right\}$ and $D_{t}^{+}(\sigma)=\left\{(s, \rho) \in D_{t}(\sigma): t \geq 0\right\}$. We refer to processes of these kinds as ambit processes, and we say that such a process is of Brownian type if $L$ is the Brownian sheet $B S$ and of shot noise type in case $L$ is a pure jump basis (i.e. $a=b=0$ in (7)).

Now suppose that $L$ is Brownian sheet and, for simplicity, that the ambit sets are homogeneous (cf. (3)-(4)) and $A_{t}(\sigma)=D_{t}(\sigma)$. Then (10) may be written

$$
\begin{aligned}
Y_{t}(\sigma)= & \mu+\int_{-\infty}^{t} \int_{\sigma-c_{t-s}^{-}}^{\sigma+c_{t-s}^{+}} g(t-s, \rho-\sigma) I_{s}(\rho) B S(\mathrm{~d} s \mathrm{~d} \rho) \\
& +\int_{-\infty}^{t} \int_{\sigma-c_{t-s}^{-}}^{\sigma+c_{t-s}^{+}} h(t-s, \rho-\sigma) J_{s}(\rho) \mathrm{d} s \mathrm{~d} \rho .
\end{aligned}
$$

In particular, if

$$
c_{t-s}^{ \pm}=c^{ \pm}(t-s)
$$

for some nonnegative constants $c^{-}$and $c^{+}$(a choice motivated in the turbulence context, see Section 5.1) then

$$
\begin{aligned}
Y_{t}(\sigma)= & \mu+\int_{-\infty}^{t} \int_{\sigma-c^{-}(t-s)}^{\sigma+c^{+}(t-s)} g(t-s, \rho-\sigma) I_{s}(\rho) B S(\mathrm{~d} s \mathrm{~d} \rho) \\
& +\int_{-\infty}^{t} \int_{\sigma-c^{-}(t-s)}^{\sigma+c^{+}(t-s)} h(t-s, \rho-\sigma) J_{s}(\rho) \mathrm{d} s \mathrm{~d} \rho .
\end{aligned}
$$

Note that if $I_{s}(\rho)$ and $J_{s}(\rho)$ are stationary processes in $s$ for fixed $\rho$ then $Y_{t}(\sigma)$ is a stationary process in $t$ for fixed $\sigma$. 


\subsection{Lagrangian dynamics}

We proceed to discuss associated questions of dynamics, for processes $X_{w}=$ $Y_{t(w)}(\sigma(w))$, as introduced in Section 2.1.

Figure 1 illustrates the dynamics of $X_{w}$ along the curve $(t(w), \sigma(w))$ for arbitrary ambit sets $A_{t(w)}(\sigma(w))$. We assume that $J=I^{2}$ (this setting is sufficient for the applications to turbulence that will be reviewed later). Further, for notational simplicity we let $\mu=0$ and we restrict consideration to the specification (14).

The process $X_{w}$ may be written as

$$
\begin{aligned}
X_{w}= & \int_{-\infty}^{t} \int_{-\infty}^{\sigma+c^{+}(t-s)} g(t-s, \rho-\sigma) I_{s}(\rho) B S(\mathrm{~d} s \mathrm{~d} \rho) \\
& +\int_{-\infty}^{t} \int_{-\infty}^{\sigma+c^{+}(t-s)} h(t-s, \rho-\sigma) J_{s}(\rho) \mathrm{d} s \mathrm{~d} \rho \\
& -\int_{-\infty}^{t} \int_{-\infty}^{\sigma-c^{-}(t-s)} g(t-s, \rho-\sigma) I_{s}(\rho) B S(\mathrm{~d} s \mathrm{~d} \rho) \\
& -\int_{-\infty}^{t} \int_{-\infty}^{\sigma-c^{-}(t-s)} h(t-s, \rho-\sigma) J_{s}(\rho) \mathrm{d} s \mathrm{~d} \rho
\end{aligned}
$$

with $(t, \sigma)=(t(w), \sigma(w))$. Continuing to argue formally, and suppressing in the notation the dependence of $t$ and $\sigma$ on $w$, we find

$$
\begin{aligned}
& \mathrm{d} X_{w}= \\
& \quad \int_{-\infty}^{t} g\left(t-s, c^{+}(t-s)\right) I_{s}\left(\sigma+c^{+}(t-s)\right) B S\left(\mathrm{~d} s \mathrm{~d}_{w}\left(\sigma+c^{+}(t-s)\right)\right) \\
& -\int_{-\infty}^{t} g\left(t-s,-c^{-}(t-s)\right) I_{s}\left(\sigma-c^{-}(t-s)\right) B S\left({\left.\mathrm{~d} s \mathrm{~d}_{w}\left(\sigma-c^{-}(t-s)\right)\right)}_{+\mathrm{d} R_{w}}\right.
\end{aligned}
$$

where

$$
\begin{aligned}
\frac{\mathrm{d} R_{w}}{\mathrm{~d} w}= & \int_{-\infty}^{t} \int_{\sigma-c^{-}(t-s)}^{\sigma+c^{+}(t-s)} \mathrm{d}_{w} g(t-s, \rho-\sigma) I_{s}(\rho) B S(\mathrm{~d} s \mathrm{~d} \rho) \\
& +\int_{-\infty}^{t} \int_{\sigma-c^{-}(t-s)}^{\sigma+c^{+}(t-s)} \mathrm{d}_{w} h(t-s, \rho-\sigma) J_{s}(\rho) \mathrm{d} s \mathrm{~d} \rho \\
& +\int_{-\infty}^{t} h\left(t-s, \sigma+c^{+}(t-s)\right) J_{s}\left(\sigma+c^{+}(t-s)\right) \mathrm{d} s \mathrm{~d}_{w}\left(\sigma+c^{+} t\right) \\
& -\int_{-\infty}^{t} h\left(t-s, \sigma-c^{-}(t-s)\right) J_{s}\left(\sigma-c^{-}(t-s)\right) \mathrm{d} s \mathrm{~d}_{w}\left(\sigma-c^{-} t\right) .
\end{aligned}
$$

Consequently, 


$$
\begin{aligned}
\frac{\left(\mathrm{d} X_{w}\right)^{2}}{\mathrm{~d} w}= & \left|\sigma^{\prime}+c^{+} t^{\prime}\right| \int_{-\infty}^{t} g^{2}\left(t-s, c^{+}(t-s)\right) J_{s}\left(\sigma+c^{+}(t-s)\right) \mathrm{d} s \\
& +\left|\sigma^{\prime}-c^{-} t^{\prime}\right| \int_{-\infty}^{t} g^{2}\left(t-s,-c^{-}(t-s)\right) J_{s}\left(\sigma-c^{-}(t-s)\right) \mathrm{d} s \\
= & \left|\sigma^{\prime}+c^{+} t^{\prime}\right| \int_{0}^{\infty} g^{2}\left(s, c^{+} s\right) J_{s}\left(\sigma+c^{+} s\right) \mathrm{d} s \\
& +\left|\sigma^{\prime}-c^{-} t^{\prime}\right| \int_{0}^{\infty} g^{2}\left(s,-c^{-} s\right) J_{s}\left(\sigma-c^{-} s\right) \mathrm{d} s
\end{aligned}
$$

We adopt the notation $\varepsilon$ for $\left(\mathrm{d} X_{w}\right)^{2} / \mathrm{d} w$.

Three special cases are of particular interest: (i) $t(w)=w, \sigma(w)=\sigma$ constant (ii) $t(w)=t$ constant, $\sigma(w)=w$ (iii) $t(w)=w, \sigma(w)=\sigma+c^{-} w$. The triangular specification (13) of the ambit set along the curves (i), (ii) and (iii) is illustrated in Figures 2-4, respectively. For these the expression (16) becomes respectively

$$
\begin{gathered}
\varepsilon_{\text {time }}(t, \sigma)= \\
\int_{0}^{\infty}\left[c^{-} g^{2}\left(s,-c^{-} s\right) J_{t-s}\left(\sigma-c^{-} s\right)+c^{+} g^{2}\left(s, c^{+} s\right) J_{t-s}\left(\sigma+c^{+} s\right)\right] \mathrm{d} s \\
\varepsilon_{\text {space }}(t, \sigma)=\int_{0}^{\infty}\left[g^{2}\left(s,-c^{-} s\right) J_{t-s}\left(\sigma-c^{-} s\right)+g^{2}\left(s, c^{+} s\right) J_{t-s}\left(\sigma+c^{+} s\right)\right] \mathrm{d} s \\
\varepsilon_{\text {Lagr }}(t, \sigma)=\left(c^{-}+c^{+}\right) \int_{0}^{\infty} g^{2}\left(s, c^{+} s\right) J_{t-s}\left(\sigma+c^{+} s\right) \mathrm{d} s
\end{gathered}
$$

In the turbulence context $\varepsilon_{\text {time }}$ is identified with the temporal energy dissipation and $\varepsilon_{\text {space }}$ is identified with the surrogate energy dissipation (cf. Section 3 ). The case (iii) corresponds to the energy dissipation obtained by following the mean flow in turbulence (cf. Section 5.1).

\subsection{Discussion}

Let $Y_{t}(\sigma)$ be an ambit spatio-temporal process of the general form (10), let $\omega(w)=(t(w), \sigma(w))$ be a curve in $\mathbb{R}^{2}$ with $t(w)$ increasing in $w$, and let

$X_{w}=\int_{A_{t}(\sigma)} g(t-s, \rho-\sigma) I_{s}(\rho) L(\mathrm{~d} s \mathrm{~d} \rho)+\int_{D_{t}(\sigma)} h(t-s, \rho-\sigma) J_{s}(\rho) \mathrm{d} s \mathrm{~d} \rho$

where again, in the notation, we have suppressed the dependence of $t$ and $\sigma$ on $w$. The following questions are of interest and do not seem answerable in any immediate fashion from existing results in the literature on spatiotemporal processes and on stochastic integration with respect to multiparameter martingales. (In the present context, key references to that literature are Cairoli and Walsh (1975), Khoshnevisan (2002), Klein and Giné (1975), Walsh (1986a,b) and Wong and Zakai (1974).) 
(i) Under what conditions, especially on the ambit sets $A_{t}(\sigma)$ and $D_{t}(\sigma)$, does the quadratic variation $[X]$ exist (in the sense of (1)).

(ii) Under what conditions, especially on the ambit sets $A_{t}(\sigma)$ and $D_{t}(\sigma)$, is it possible meaningfully to define the differential $\mathrm{d} X$.

(iii) Related to (ii), what meaning should be given to an expression like

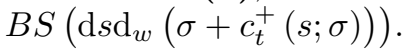

(iv) Supposing $L=B S$ (the Brownian sheet), when is $(\mathrm{d} X)^{2}=\mathrm{d}[X]$ (or, otherwise put, when is $\left.[X]_{w}=\int_{0}^{w}\left(\mathrm{~d} X_{s}\right)^{2}\right)$.

(v) When is $X$ a linear combination of semimartingales.

For the particular specification of $X$ considered in Section 2.4, we have argued as if these questions had been positively resolved. In fact, under mild assumptions on $g, h, I$ and $J$ the manipulations in that Section can be verified by direct calculations (details to be given elsewhere).

\section{Some background on turbulence}

There is no generally accepted definition of what should be called a turbulent flow. Turbulent flows are characterized by low momentum diffusion, high momentum convection, and rapid variation of pressure and velocity in space and time. Flow that is not turbulent is called laminar flow. The non-dimensional Reynolds number $R$ characterizes whether flow conditions lead to laminar or turbulent flow. Increasing the Reynolds number increases the turbulent character and the limit of infinite Reynolds number is called the fully developed turbulent state.

Turbulence as part of hydrodynamics is governed by the Navier-Stokes equation which has been known since 1823. Its non-linear and non-local character does so far not allow to describe the wide range of turbulent phenomena from basic principles. Consequently, a great deal of phenomenological models have emerged that are based on and designed for certain aspects of turbulent dynamics. Most of these models can be classified according to the physical observable they address (see Section 4). The most prominent observables are the velocity field and the energy dissipation process.

In general, turbulence concerns the dynamics in a fluid flow of the threedimensional velocity vector $\mathbf{u}(\mathbf{r}, t)=\left(u_{x}(\mathbf{r}, t), u_{y}(\mathbf{r}, t), u_{z}(\mathbf{r}, t)\right)$ as a function of position $\mathbf{r}=(x, y, z)$ and time $t$. A derived quantity is the energy dissipation, defined as

$$
\varepsilon(\mathbf{r}, t) \equiv \frac{\nu}{2} \sum_{i, j=x, y, z}\left(\partial_{i} u_{j}(\mathbf{r}, t)+\partial_{j} u_{i}(\mathbf{r}, t)\right)^{2}
$$

describing the loss of kinetic energy due to friction forces characterized by the viscosity $\nu$.

A pedagogical valuable illustration of a turbulent flow can be gained from the Kolmogorov cascade (Frisch (1995)). In this representation kinetic energy is injected into the flow at large scales through large scale forcing. Non-linear 
effects redistribute the kinetic energy towards smaller scales. This cascade of energy stops at small scales where dissipation transforms kinetic energy into heat. It is traditional to call the large scale $L$ of energy input the integral scale and the small scale $\eta$ of dissipation the dissipation scale or Kolmogorov scale. With increasing Reynolds number the fraction $L / \eta$ increases, giving space for the so called inertial range $\eta \ll l \ll L$ where turbulent statistics are expected to have some universal character.

The resolution of all dynamically active scales in experiments is at present not achievable for the full three-dimensional velocity vector. Most experiments measure a time-series of one component $u$ (in direction of the mean flow) of the velocity vector at a fixed single location $\mathbf{r}_{0}$ (in the stochastic framework we denote the spatial location by $\sigma$ ). Based on this restriction one defines the temporal energy dissipation

$$
\varepsilon_{\text {time }}\left(\mathbf{r}_{0}, t\right) \equiv \frac{15 \nu}{\bar{u}^{2}}\left(\frac{\mathrm{d} u\left(\mathbf{r}_{\mathbf{0}}, t\right)}{\mathrm{d} t}\right)^{2},
$$

where $\bar{u}$ denotes the mean velocity.

In going from (21) to (22) one assumes the flow to be stationary, homogeneous and isotropic. In this case (21) may be approximated as (Elsner and Elsner (1996))

$$
\varepsilon_{\text {space }}(\mathbf{r}, t) \equiv 15 \nu\left(\frac{\partial u(\mathbf{r}, t)}{\partial x}\right)^{2}
$$

which is believed to have similar statistical properties as the true energy dissipation at not too small scales. Discrepancies appear at small scales and are termed surrogacy effects. In particular, the autocorrelation function of the surrogate energy dissipation (23) shows an additional increase at small time scales (Cleve et al (2003)).

The transformation of the spatial derivative in (23) to the temporal derivative in (22) is performed under the assumption of Taylor's Frozen Flow Hypothesis (Taylor (1938)) which states that spatial structures of the flow are predominantly swept by the mean velocity $\overline{\mathbf{u}}$ without relevant distortion. Under this hypothesis, widely used in analyzing turbulent time series, spatial increments along the direction of the mean flow (in direction $x$ ) are expressed in terms of temporal increments

$$
u_{t+s}(\mathbf{r})-u_{t}(\mathbf{r})=u_{t}(\mathbf{r}-\overline{\mathbf{u}} s)-u_{t}(\mathbf{r}) .
$$

Remark 2. The temporal energy dissipation (22) is expected to approximate the true energy dissipation (21) for stationary, homogeneous and isotropic flows. Nevertheless, the temporal energy dissipation contains for all flow conditions important statistical information about the turbulent velocity field. 


\section{Turbulence phenomenology}

The statistical analysis of a great variety of time series has revealed a number of universal stylized facts of homogeneous and isotropic turbulent flows. Here we restrict the discussion to the so-called intermittency and to the statistics associated with the Kolmogorov variable, leaving aside, among others, the important characterization of turbulent statistics in terms of scaling relations (Meneveau and Sreenivasan (1991) and Sreenivasan and Antonia (1997) and references therein). Scaling relations are expected to hold for fully developed turbulent flows while being hard to detect for small and moderate Reynolds number flows. Intermittency and universality of the statistics associated to the Kolmogorov variable are found for a much wider range of Reynolds numbers (Castaing et al (1990), Vincent and Meneguzzi (1991), Barndorff-Nielsen et al (2004), Stolovitzky et al (1992), Zhu et al (1995) and Hosokawa et al (1994)).

\subsection{Intermittency}

Since the pioneering work of Kolmogorov (1962) and Obukhov (1962), intermittency of the turbulent velocity field is of major interest in turbulence research. From a probabilistic point of view, intermittency refers, in particular, to the increase of the non-Gaussian behaviour of the probability density function (pdf) of velocity increments with decreasing scale. A typical scenario is characterized by an approximate Gaussian shape for the large scales, turning to exponential tails for the intermediate scales and stretched exponential tails for dissipation scales (Castaing et al (1990) and Vincent and Meneguzzi (1991)).

It was reported in Barndorff-Nielsen et al (2004) that the evolution of the pdf of velocity increments for all amplitudes and all scales can be described within one class of analytically tractable distributions, the normal inverse Gaussian (NIG) distributions. This class of distributions equals the family of possible distributions at time $t=1$ of the NIG Lévy process, which is defined as Brownian motion with drift subordinated by the inverse Gaussian Lévy process, i.e. the Lévy process of first passage times to constant levels of (another) Brownian motion. The Appendix provides a brief summary of the definition and properties of NIG laws.

The NIG laws and associated processes have found widespread application, particularly in finance, see for instance Barndorff-Nielsen (1998a,b), Barndorff-Nielsen and Shephard (2001), Barndorff-Nielsen and Shephard (2006), Øigård et al (2005), Corsi et al (2005), Carr et al (2003), Forsberg (2002), Lindberg (2005), Eberlein and Prause (2002) and further references there, cf. also Shiryaev (1999) and Cont and Tankov (2004).

Figure 5 shows, as an example, the log densities of velocity increments $\Delta u_{s}=u_{t+s}-u_{t}$ measured in the atmospheric boundary layer for various time scales $s$. The solid lines denote the approximation of these densities within 
the class of NIG distributions. NIG distributions fit the empirical densities equally well for all time scales $s$.

A subsequent analysis of the observed parameters of the NIG distributions from many, widely different data sets with Reynolds numbers ranging from $R_{\lambda}=80$ up to $R_{\lambda}=17000$ (where $R_{\lambda}$ is the Taylor based Reynolds number, see below) led to the formulation of a key universality law (Barndorff-Nielsen et al (2004)): The temporal development of a turbulent velocity field has an intrinsic clock which depends on the experimental conditions but in terms of which the one-dimensional marginal distributions of the velocity differences become independent of the experimental conditions. Figure 6 provides an empirical validation of this. As a consequence, the collapse of pdf's immediately resulted in a substantially wider and more general reformulation of the concept of Extended Self Similarity (Benzi et al (1993)) in terms of a stochastic equivalence class. For details we refer to Barndorff-Nielsen et al (2004).

\subsection{Kolmogorov's refined hypotheses}

In 1962, Kolmogorov published two hypotheses (usually refered to as K62) about a quantity $V$ that combines velocity increments, being a large scale quantity, and the energy dissipation, being a small scale quantity. The first hypothesis states that the pdf of the stochastic variable

$$
V_{r}=\frac{\Delta u_{t}(r)}{\left(r \varepsilon_{r}\right)^{1 / 3}}
$$

depends, for $r \ll L$, only on the local Reynolds number

$$
\operatorname{Re}_{r}=r\left(r \varepsilon_{r}\right)^{1 / 3} / \nu .
$$

Here,

$$
\Delta u_{t}(r)=u_{t}(x+r, y, z)-u_{t}(x, y, z)
$$

denotes the increment of one component of the velocity vector at scale $r$ and $r \varepsilon_{r}$ is the integrated energy dissipation over a domain of linear size $r$

$$
\varepsilon_{r}=\frac{1}{r} \int_{x_{0}-r / 2}^{x_{0}+r / 2} \varepsilon(\mathbf{r}, t) \mathrm{d} x .
$$

The second hypothesis states that, for $\operatorname{Re}_{r} \gg 1$, the pdf of $V_{r}$ does not depend on $\mathrm{Re}_{r}$, either, and is therefore universal.

Note the unusual power $1 / 3$ in (25). An immediate thinking would have expected power $1 / 2$.

Although, for small $r$, an additional $r$ dependence of the pdf of $V_{r}$ has been observed (Stolovitzky et al (1992)), the validity of several aspects of K62 has been verified experimentally and by numerical simulation of turbulence (Stolovitzky et al (1992), Zhu et al (1995), Hosokawa et al (1994) and 
Stolovitzky and Sreenivasan (1994)). In particular it has been shown that the conditional densities $p\left(V_{r} \mid r \varepsilon_{r}\right)$ become independent of $r \varepsilon_{r}$ for a certain range of scales $r$ within the inertial range. However, the universality of the distribution of $V$ has not been verified in the literature. In this respect, it is important to note that the experimental verification of the Kolmogorov hypotheses is, with reasonable resolution of scales, restricted to temporal statistics and as such relies on the use of the temporal energy dissipation (22) instead of the true energy dissipation (21).

We take up the discussion of the Kolmogorov variable $V$ in Section 5.2.

\section{Stochastic modelling of turbulent velocity fields}

The modelling framework we propose for the velocity field specifies this as an ambit process and incorporates the energy dissipation, also in the form of an ambit process, as a building block. As we shall discuss, basic stylized facts of turbulent statistics are captured by the model without specifying the degrees of freedom in all detail.

Remark 3. For the energy dissipation, discrete cascade processes are one of the most basic and successful models (Meneveau and Sreenivasan (1991), Jouault et al (1999), Jouault et al (2000) and Cleve and Greiner (2000)). However, these models lack translational invariance and moreover, they introduce an artifical and discrete hierarchy of scales. To overcome these drawbacks, ambit

processes can be used as continuous and translation invariant generalisations of discrete cascade models (cf. Section 5.3).

\subsection{A spatio-temporal modelling framework}

We propose to model one component of the velocity vector in homogeneous and stationary turbulence as in (14)

$$
\begin{aligned}
u_{t}(\sigma)= & \mu+\int_{-\infty}^{t} \int_{\sigma-c^{-}(t-s)}^{\sigma+c^{+}(t-s)} g(t-s, \rho-\sigma) I_{s}(\rho) B S(\mathrm{~d} s \mathrm{~d} \rho) \\
& +\beta \int_{-\infty}^{t} \int_{\sigma-c^{-}(t-s)}^{\sigma+c^{+}(t-s)} h(t-s, \rho-\sigma) J_{s}(\rho) \mathrm{d} s \mathrm{~d} \rho
\end{aligned}
$$

where $\mu$ and $\beta$ are constants, $c^{+}$and $c^{-}$are positive constants and we assume $J=I^{2}$, which is sufficiently general in the turbulence context. Here we adopt the notation $u$ (instead of $Y$ ) for the velocity as is customary in the physics literature. The specific choice of a triangular ambit set corresponds to a constant maximum speed for information to arrive at a given site $(\sigma, t)$. In this simple set-up the influence of an event sitting at $\rho<\sigma$ or $\sigma<\rho$ is experienced at $\sigma$ with a delay of $(\sigma-\rho) / c^{-}$or $(\rho-\sigma) / c^{+}$, respectively. The difference in 
the propagation velocities for $\sigma>\rho$ and $\sigma<\rho$ is due to the presence of a mean velocity. In general, interactions in the flow are due to pressure fluctuations traveling with the speed of sound $c$ and interactions that are sweeping with the flow. Here we only deal with the simplest case where the sweeping velocity is assumed to be the mean velocity $\bar{u}>0$. In this case

$$
c^{+}=c-\bar{u}, \quad c^{-}=c+\bar{u}
$$

In this definition, density fluctuations are taken into account which corresponds to compressible flows. The ratio $\bar{u} / c$ is called the Mach number.

For incompressible flows, density fluctuations are neglected and this is encompassed by the model (29) in setting

$$
c^{+}=0, \quad c^{-}=\bar{u} .
$$

The mean velocity $\bar{u}$ is a free parameter of the model related to $\mu$ by

$$
\mu=\bar{u}-\beta c_{1}(J) \int_{0}^{\infty} \int_{-c^{-} s}^{c^{+} s} h(s, \rho) \mathrm{d} s \mathrm{~d} \rho .
$$

In the setting of stochastic differential equations of the Brownian semimartingale type $(29)$ the quantity $\left[\mathrm{d} u_{t}(\sigma)\right]^{2} / \mathrm{d} t(17)$ is the natural analogue of the squared first order derivative of the velocity, which in the classical formulation is taken to express the temporal local energy dissipation (22) (up to a constant pre-factor). In a similar reasoning, $\left[\mathrm{d} u_{t}(\sigma)\right]^{2} / \mathrm{d} \sigma(18)$ may be identified with (23) (up to a constant pre-factor). In both cases, the local energy dissipation is independent of the second term in (29) which, importantly, allows to choose the function $h$ and the constant $\beta$ in (29) independently of the energy dissipation process.

The intermittency of the model, i.e. its non-Gaussian statistics, arises from both terms in (29). In particular the third order cumulant results in a polynomial of third order in $\beta$. Here we do not present the results for the full cumulant function of velocity increments. We rather specify the intermittent and turbulent character of the model in terms of the Taylor based Reynolds number (Frisch (1995)) defined as

$$
R_{\lambda}=\frac{c_{2}(u)}{\nu \sqrt{\mathrm{E}\left\{\varepsilon_{\text {space }}\right\}}} .
$$

Using (29) and (18), we calculate this most prominent characteristic of turbulence to be

$$
R_{\lambda}=\frac{1}{\nu}\left(G_{1}+G_{2}(\beta)\right)
$$

where

$$
G_{1}=\sqrt{c_{1}(J)} \frac{\int_{0}^{\infty} \int_{-c^{-} s}^{c^{+} s} g^{2}(s, \rho) \mathrm{d} s \mathrm{~d} \rho}{\sqrt{\int_{0}^{\infty}\left(g^{2}\left(s,-c^{-} s\right)+g^{2}\left(s, c^{+} s\right)\right) \mathrm{d} s}}
$$


and $G_{2}(\beta)=\beta^{2} G_{2}$ where

$$
G_{2}=\frac{\int_{0}^{\infty} \int_{0}^{\infty} \int_{-c^{-} s}^{c^{+} s} \int_{-c^{-} s^{\prime}}^{c^{+} s^{\prime}} h(s, \rho) h\left(s^{\prime}, \rho^{\prime}\right) \operatorname{Cov}\left\{J_{s}(\rho), J_{s^{\prime}}\left(\rho^{\prime}\right)\right\} \mathrm{d} s \mathrm{~d} s^{\prime} \mathrm{d} \rho \mathrm{d} \rho^{\prime}}{\sqrt{c_{1}(J)} \sqrt{\int_{0}^{\infty}\left(g^{2}\left(s,-c^{-} s\right)+g^{2}\left(s, c^{+} s\right)\right) \mathrm{d} s}},
$$

Cov indicating covariance.

The first term (35) is independent of the weight function $\beta h$. Therefore we are able to increase $R_{\lambda}$ by manipulating $\beta$ and/or the function $h$ without changing the statistics of the energy dissipation. In other words, the level of turbulence can be increased independently of the energy dissipation process. This type of behaviour has been observed for flows with strong shear where the intermittency of the velocity field (measured in terms of structure functions) shows an enhanced degree while the energy dissipation behaves in a universal fashion (Casciola et al (2001)).

\subsection{A temporal modelling framework}

The spatio-temporal $(1+1)$-dimensional model $(29)$ and its generalization to higher dimensional modelling provides the general modelling framework for the turbulent velocity field. For a preliminary verification of the proposed modelling framework with experimental data we restrict ourselves to purely temporal statistics at a fixed spatial position $\sigma$, which are by now the type of data that are accessible with reasonable quality. For mathematical simplicity, we define a purely temporal version of (29) as

$$
u_{t}=\mu+\int_{-\infty}^{t} g(t-s) I_{s} \mathrm{~d} B_{s}+\beta \int_{-\infty}^{t} g(t-s) J_{s} \mathrm{~d} s,
$$

where $B$ denotes Brownian motion. This model is in fact a limiting case of (29) with $h=g$, for $c^{-}=c^{+}=c / 2 \rightarrow \infty$ and $g(s, \rho)=c^{-1} s^{-1+c} g(s)$. The statistical properties of (37) are reported in more detail in BarndorffNielsen and Schmiegel (2005) where it was shown that a considerable part of its statistics are mediated by the structure of the model without specifying the intermittency $J$ and the weight function $g$ in all details. In the following we review the validation of the model (37) concerning the evolution of the density of velocity increments across time scales and the experimental verification of the statistics of the Kolmogorov variable.

In the setting of the model (37), the local energy dissipation can be identified with $\left[\mathrm{d} u_{t}\right]^{2} / \mathrm{d} t=J_{t}$ and consequently the quadratic variation $[u]_{t}$ is the stochastic analogue of the integrated energy dissipation.

As for the spatio-temporal model (29), the energy dissipation process does not depend on $\beta$. The constant $\beta$ introduces a non-vanishing skewness in accordance with Kolmogorov's famous $4 / 5$-the law (Kolmogorov (1941)). For the calculations below and for simulations we set $\beta=0$, for convenience. This restriction does not essentially alter the results we derived by simulations. 


\section{Temporal model and shape dynamics}

As mentioned earlier, the density of empirical velocity increments evolves from heavy tails at small time scales $s$ towards an approximate Gaussian shape at large scales $s$, in a manner that is well described within the class of NIGdistributions.

In comparing this to properties of the model (37) the first thing to note is that under (37) the asymptotic law of $u_{t}-u_{0}$ for $t \rightarrow \infty$ will not be Gaussian unless the intermittency field $I$ is deterministic. This is in accordance with experimental findings, as illustrated by Figure 7 which shows the estimated (by maximum likelihood) asymmetry and steepness parameters $\chi$ and $\xi$ of the fitted NIG laws, plotted in the NIG shape triangle (see Appendix). Note that the normal law occurs as a limiting case near $(\chi, \xi)=(0,0)$. The data are from the atmospheric boundary layer (see also Figure 5).

To quantify the non-Gaussian character of the density of velocity increments in the model (37) we first focus on the standardized fourth order cumulant $\bar{c}_{4}$ which, in the absence of skewness $\beta=0$, is the first order that distinguishes between a Gaussian shape and a heavytailed distribution. A specific result can be obtained by setting

$$
g(t)=e^{-\gamma t}
$$

and assuming $J$ to be of Ornstein-Uhlenbeck-type, i.e.

$$
J_{t}=\int_{-\infty}^{t} e^{-\lambda(t-s)} \mathrm{d} L_{s}
$$

where $L$ is the inverse Gaussian Lévy process. For brevity a process $J$ of this form is refered to as an OU-IG process.

The parameters $\lambda$ and $\gamma$ control the autocorrelation functions of $J$ and $u$, respectively. In this case we obtain

$$
\lim _{t \rightarrow \infty} \bar{c}_{4}\left(u_{t}-u_{0}\right)=\frac{3 c_{2}\left(L_{1}-L_{0}\right)}{2 c_{1}\left(L_{1}-L_{0}\right)^{2}} \frac{\gamma \lambda}{2 \gamma+\lambda} .
$$

and

$$
\lim _{t \rightarrow 0} \bar{c}_{4}\left(u_{t}-u_{0}\right)=\frac{3 c_{2}\left(L_{1}-L_{0}\right)}{2 c_{1}\left(L_{1}-L_{0}\right)^{2}} \lambda .
$$

The heaviness of the tails of the pdf of velocity increments increases with increasing $\lambda$, i.e. with a faster decrease of correlations of the local energy dissipation. Qualitatively, the same behaviour is observed for turbulent flows where the heaviness of the tails of the pdf of velocity increments increases with increasing Reynolds number and with increasing intermittency exponent $\mu_{2}$ (Cleve et al (2004)), defined as $\mathrm{E}\left\{\varepsilon_{0} \varepsilon_{t}\right\} \sim t^{-\mu_{2}}$. (Due to this power-law behaviour, the assumption of $\varepsilon(=J)$ following an OU-IG process, for which $\mathrm{E}\left\{\varepsilon_{0} \varepsilon_{t}\right\}=c_{2}\left(L_{1}-L_{0}\right)[2 \lambda]^{-1} e^{-\lambda t}+c_{1}\left(L_{1}-L_{0}\right)^{2} \lambda^{-2}$, is not a quite realistic 
approach for modelling the local energy dissipation. We come back to this point in Section 5.3.)

The corresponding results for moderate time scales are only accessible through numerical simulation. For the simulations we set $\beta=0$ in (37) and model $J$ as an OU-IG-process. Figure 8 shows the evolution of the probability densities of the simulated increments $u_{t}-u_{0}$ for various time scales $t$. We clearly observe heavy tails for the small scales and an approximately Gaussian shape for the large scales. The solid lines denote the approximation of the densities within the class of NIG-distributions. The densities of $u_{t}-u_{0}$

qualitatively display the empirical findings about the evolution across scales of turbulent velocity increments shown in Figure 5.

\section{Temporal model and K62}

As the second validation of the model (37) we briefly discuss K62 and its experimental verification. The original definition of $V$ in (25) relates to spatial statistics which are not accessible in experiments. Therefore, the experimental verification of $\mathrm{K} 62$ has been performed in terms of temporal analysis. In the temporal model (37), the Kolmogorov variable may be defined as

$$
V_{t}=\frac{u_{t}-u_{0}}{\left\{\bar{u}[u]_{t}\right\}^{1 / 3}} .
$$

The introduction of the mean velocity $\bar{u}$ turns $V_{t}$ into a non-dimensional stochastic process.

The most important property of $V$ concerns its conditional statistics. $\mathrm{Nu}-$ merous investigations of turbulent data sets show that the conditional densities $p\left(V_{t} \mid[u]_{t}\right)$ become independent of $[u]_{t}$ for not too small $t$. Within the model (37) this observation is confirmed to high accuracy by simulations done with the same parameters as for the simulation of the densities of velocity increments in Figure 8. Figures 9-10 show the conditional densities $p\left(V_{t} \mid[u]_{t}\right)$ for $t=2$ and $t=16$ and various values of $[u]_{t}$. For small $t$, the conditional densities strongly depend on $[u]_{t}$. With increasing time scale $t$, the dependence gets smaller and for large enough $t(t \approx 16$ in our simulation), the conditional densities do not depend on $[u]_{t}$. This independence also holds for the larger time scales $t>16$ (not shown here). These findings agree well with results reported for the turbulent velocity field in Stolovitzky et al (1992), Zhu et al (1995) and Stolovitzky and Sreenivasan (1994), and they reveal the gist of K62.

The exponent $1 / 3$ in the definition of the Kolmogorov Variable $V$ in (42) has been introduced by Kolmogorov for dimensional reasons (threedimensional space). In order to give an impression about the peculiarity of $1 / 3$ we define

$$
V_{\alpha, t}=\frac{u_{t}-u_{0}}{\left\{\bar{u}[u]_{t}\right\}^{\alpha}} .
$$


which coincides with (42) for $\alpha=1 / 3$. To assess the question of how much the independence of the conditional densities $p\left(V_{t} \mid[u]_{t}\right)$ on $[u]_{t}$ depends on the specific choice $\alpha=1 / 3$ we analyse the dependence of the second-order conditional cumulants $c_{2}\left(V_{\alpha, t} \mid[u]_{t}\right)$ on $[u]_{t}$ for different values of $\alpha$. Figure 11 compares $c_{2}\left(V_{\alpha, t} \mid[u]_{t}\right)$ for $\alpha=1 / 2$ and $\alpha=1 / 3$. The conditional cumulants are estimated from simulations with the same parameters as used for the simulation of velocity increments in Figure 8. For $\alpha=1 / 2$ the conditional cumulants considerably decrease with increasing $[u]_{t}$. For $\alpha=1 / 3$ the conditional cumulants stay roughly constant. For the moment, we have no explanation for why the model seems to be adapted to the exponent $1 / 3$ (or at least to an exponent close to $1 / 3$ ).

\subsection{The energy dissipation process}

The basic ingredient of the model for the turbulent velocity is the intermittency process $J$. For the temporal model (37), $J$ coincides with the temporal energy dissipation $\varepsilon_{\text {time }}$. For the more general spatio-temporal model (29), the energy dissipation is expressed as an integral over the weighted $J$ process (see Section 2.4). In the following, we discuss a particular model for the energy dissipation process $\varepsilon$ that is along the line of ambit processes (Schmiegel et al (2006), Barndorff-Nielsen and Schmiegel (2004), Schmiegel et al (2004), Schmiegel (2005a)).

We model the energy dissipation process as an ambit process of the exponential form

$$
\varepsilon_{t}(\sigma)=\exp \left\{\int_{C_{t}(\sigma)} f(|t-s|,|\sigma-\rho|) L(\mathrm{~d} s \mathrm{~d} \rho)\right\},
$$

where $L$ is a homogeneous and factorisable Lévy basis and $f$ is an integrable deterministic function. Then we have the fundamental relation

$$
\mathrm{E}\left\{\exp \left\{\int_{C} f(c) L(\mathrm{~d} c)\right\}\right\}=\exp \left\{\int_{C} \mathrm{~K}[f(c)] \mathrm{d} c\right\},
$$

where $\mathrm{K}$ denotes the cumulant function of $L(\mathrm{~d} c)$, defined by

$$
\ln \mathrm{E}\{\exp \{\xi L(\mathrm{~d} c)\}\}=\mathrm{K}[\xi] \mathrm{d} c .
$$

The usefulness of (45) is obvious: it permits explicit calculation of the correlation function of the integrated and $f$-weighted noise field $L(\mathrm{~d} c)$ once the cumulant function $\mathrm{K}$ is known.

The generality of the model (44) is based on the possibility of choosing the constituents of the process $\varepsilon_{t}(\sigma)$ independently. The available degrees of freedom are an arbitrary infinitely divisible law for the Lévy basis $L$, the deterministic function $f$ and the shape of the family $C$ of ambit sets. 
Despite its generality, the model is tractable enough to yield explicit expressions for arbitrary $n$-point correlations $\mathrm{E}\left\{\varepsilon_{t_{1}}\left(\sigma_{1}\right) \cdot \ldots \cdot \varepsilon_{t_{n}}\left(\sigma_{n}\right)\right\}$ in closed form.

Here, we focus on two-point correlators of order $\left(n_{1}, n_{2}\right)$, defined as

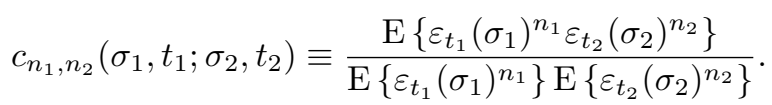

In the following we set $f \equiv 1$. This choice of the weight function $f$ is motivated by the fact that two-point correlators obtained from a variety of turbulent data sets show the property of self-scaling (see below). Moreover, the freedom of choosing an arbitrary shape of the ambit set $C$ is sufficient to model a wide range of two-point correlators of order $(1,1)$ which are of primary interest in the present context.

Using (45), it is straightforward to show that

$$
c_{n_{1}, n_{2}}\left(\sigma_{1}, t_{1} ; \sigma_{2}, t_{2}\right)=\exp \left\{\overline{\mathrm{K}}\left[n_{1}, n_{2}\right] \int_{C_{t_{1}}\left(\sigma_{1}\right) \cap C_{t_{2}}\left(\sigma_{2}\right)} \mathrm{d} \sigma \mathrm{d} t\right\},
$$

with the abbreviation $\overline{\mathrm{K}}\left[n_{1}, n_{2}\right]=\mathrm{K}\left[n_{1}+n_{2}\right]-\mathrm{K}\left[n_{1}\right]-\mathrm{K}\left[n_{2}\right]>0$ (as follows from the Minkowski inequality). The important point here is the fact that the exponent in (48) factorizes into the Euclidean volume of the overlap of the two ambit sets times a factor depending only on the order $\left(n_{1}, n_{2}\right)$. Thus we are able to rewrite (48) as a self-scaling relation of two point correlators of orders $\left(n_{1}, n_{2}\right)$ and $\left(m_{1}, m_{2}\right)$ (Schmiegel (2005a))

$$
c_{n_{1}, n_{2}}\left(\sigma_{1}, t_{1} ; \sigma_{2}, t_{2}\right)=c_{m_{1}, m_{2}}\left(\sigma_{1}, t_{1} ; \sigma_{2}, t_{2}\right)^{k\left[m_{1}, m_{2} ; n_{1}, n_{2}\right]}
$$

with the abbreviation

$$
k\left[m_{1}, m_{2} ; n_{1}, n_{2}\right]=\frac{\overline{\mathrm{K}}\left[n_{1}, n_{2}\right]}{\overline{\mathrm{K}}\left[m_{1}, m_{2}\right]},
$$

called the self-scaling exponent.

The self-scaling relation (49) implies that correlators of arbitrary order $\left(n_{1}, n_{2}\right)$ are determined by the correlator of order $(1,1)$ and the knowledge of the self-scaling exponents $k$ of all orders. Note that the self-scaling exponents $k$ only depend on the Lévy basis $L$.

For a given Lévy basis $L$ it is possible to extract the shape of the ambit set directly from two-point correlators of order $(1,1)$ which are accessible in experiments. For that we assume the ambit set $C_{t}(\sigma)$ to be of the form

$$
C_{t}(\sigma)=\{(\rho, s): t-T<s<t, \rho \in[\sigma-q(s-t+T), \sigma+q(s-t+T)]\}
$$

where the function $q(s)$, defined on $[0, T]$ is nonnegative and decreasing. The constant $T$ introduces a decorrelation time for the energy dissipation process. 
We further assume $q$ and its inverse $q^{(-1)}$ to be differentiable. In this case it is easy to give necessary and sufficient conditions on spatial two-point correlators of order $(1,1)$ to be modelled by the Ansatz (44). From (48) it follows that

$$
\begin{aligned}
\frac{\partial}{\partial l} \ln c_{1,1}(\sigma, t ; \sigma+l, t) & =\overline{\mathrm{K}}[1,1] \frac{\partial}{\partial l}\left(2 \int_{0}^{q^{(-1)}(l / 2)}(q(s)-l / 2) \mathrm{d} s\right) \\
& =-\overline{\mathrm{K}}[1,1] q^{(-1)}(l / 2)
\end{aligned}
$$

and

$$
\frac{\partial^{2}}{\partial l^{2}} \ln c_{1,1}(\sigma, t ; \sigma+l, t)=-\frac{1}{2} \overline{\mathrm{K}}[1,1] \frac{\partial}{\partial l} q^{(-1)}(l / 2) .
$$

Thus, the Ansatz (44) together with a decreasing boundary $q(t)>0$ is able to model any twice differentiable spatial two point correlator that has the properties

$$
\frac{\partial}{\partial l} \ln c_{1,1}(\sigma, t ; \sigma+l, t)<0
$$

and

$$
\frac{\partial^{2}}{\partial l^{2}} \ln c_{1,1}(\sigma, t ; \sigma+l, t)>0 .
$$

Relation (52) has been applied to turbulent data in Schmiegel et al (2004) where the shape of the ambit set has been extracted from scaling two-point correlators. As a consequence the higher order correlators are fixed and the three-point correlators have been successfully compared to experimental data.

In the temporal set-up (37) the intermittency process $J$ is identified with the local energy-disspation $\varepsilon$ and as such directly accessible to turbulent data analysis. For the more general spatio-temporal model (14) correlators of the energy-disspation can be expressed as weighted integrals over the correlators of the intermittency process $J$ and as such can be modelled by suitably adapting the weight function $g$ and the statistics of $J$.

\section{Modelling tumour growth}

The potential of processes of the type (44) for modelling a certain, well defined correlation structure may also be useful for modelling tumour dynamics (Schmiegel (2005b) and Jensen et al (2006)). The object of interest in that context is the star-shaped approximation of planar tumour tissue characterized by a radius function

$$
R_{t}(\phi)=\max \left\{R: \mathbf{c}_{0}+R \mathbf{e}_{\phi} \in S_{t}\right\}
$$

where $S_{t}$ denotes the two-dimensional domain occupied by the tumour at time $t, \mathbf{c}_{\mathbf{0}}$ denotes the centre of mass of the tumour at time $t=0$ and $\mathbf{e}_{\phi}$ is the unit vector in direction $\phi \in[0,2 \pi]$. 
Tumour profiles show structures at very different scales with strongly localized outbursts of different size. Due to the unrestricted growth of the tumour in in vitro experiments we can expect the profiles to be statistically isotropic. A comparison of these star-shaped profiles with the original profiles as observed in the experiment (Brú et al (1998)) shows that (56) approximates the growing tumour to a high accuracy. For the star-shaped approximation, we neglect details of the tumour profiles where small regions of non-tumour tissue are surrounded by tumour cells.

For the stochastic modelling of profiles we normalize the radial function

$$
r_{t}(\phi) \equiv \frac{R_{t}(\phi)}{\mathrm{E}\left\{R_{t}(\phi)\right\}},
$$

where $\mathrm{E}\left\{R_{t}(\phi)\right\}$ is the mean radius at time $t$, assumed to be independent of $\phi$. Thus, $\mathrm{E}\left\{r_{t}(\phi)\right\}=1$ for all times $t$. For the estimation of expectations, we perform spatial averaging.

Spatial correlators of star shaped tumour profiles have the form (Schmiegel $(2005 b))$

$$
\ln \left(c_{n_{1}, n_{2}}(t, \phi ; t, \phi+\Delta \phi)\right)=d_{n_{1}, n_{2}}(t) f_{t}(\Delta \phi) \mathbf{1}_{\left[0, \phi_{0}(t)\right]}(\Delta \phi)+b_{n_{1}, n_{2}}(t) \cos (\Delta \phi)
$$

where the critical angle $\phi_{0}(t)$ confines the valitidity of the cosine behaviour for $\Delta \phi>\phi_{0}$. For $\Delta \phi<\phi_{0}$ deviations from the cosine behaviour occur and are denoted by $f_{t}$. The factors $d$ and $b$ are independent of $\Delta \phi$ and depend only on the order $\left(n_{1}, n_{2}\right)$.

To account for the particular correlation structure (58) we propose an exponential ambit process of the type

$$
\begin{aligned}
r_{t}(\phi)= & \exp \left\{a(t) \int_{t-T(t)}^{t-t_{0}(t)} \int_{\phi-\pi}^{\phi+\pi} \cos \left(\phi-\phi^{\prime}\right) B S\left(\mathrm{~d} t^{\prime} \mathrm{d} \phi^{\prime}\right)\right. \\
& \left.+h(t) \int_{t-t_{0}(t)}^{t} \int_{\phi-q_{t}\left(t^{\prime}-t+t_{0}(t)\right)}^{\phi+q_{t}\left(t^{\prime}-t+t_{0}(t)\right)} B S\left(\mathrm{~d} t^{\prime} \mathrm{d} \phi^{\prime}\right)\right\},
\end{aligned}
$$

with cyclic definition in the angle and where $B S$ is a Brownian sheet. The first term on the right hand side of (59) is responsible for the validity of the cosine law (second term in (58)) and the second term on the right hand side of (59) is associated with the deviations from the cosine law at small angular distances. We call the first term in the exponent on the right hand side of (59) the large scale term and the second term the small scale term.

The ambit set associated with the large scale term is a rectangle of the form $\left[t-T(t), t-t_{0}(t)\right] \times[\phi-\pi, \phi+\pi]$. The deterministic function $T(t)$ can be interpreted as the decorrelation time of the radius process and $t_{0}(t)$ expresses the decorrelation time of the small scale term.

The ambit set associated with the small scale term is assumed to be determined by a deterministic and monotonically decreasing function $q_{t}$ defined 
on $\left[0, t_{0}(t)\right]$ and satisfying $q_{t}\left(t_{0}(t)\right)=0$. These two parts of the ambit set are weighted differently according to the deterministic functions $a(t) \cos \left(\phi-\phi^{\prime}\right)$ and $h(t)$ for the large scale term and the small scale term, respectively.

Within the modelling framework (59) the two point correlators are of the specific form (58) where

$$
b_{n_{1}, n_{2}}(t)=n_{1} n_{2} a(t)^{2} \pi\left(T(t)-t_{0}(t)\right)
$$

and the small scale amplitude $d_{n_{1}, n_{2}}(t)$ has the form

$$
d_{n_{1}, n_{2}}(t)=n_{1} n_{2} h(t)^{2}
$$

and we identify $f_{t}$ by

$$
f_{t}(\Delta \phi)=V_{t}(\Delta \phi)=\int_{0}^{q_{t}^{(-1)}(\Delta \phi / 2)}\left(2 q_{t}(s)-\Delta \phi\right) \mathrm{d} s
$$

where $V_{t}(\Delta \phi)$ is the Euclidean volume of the overlap of the ambit sets of the small scale terms separated by the angular distance $\Delta \phi$. The critical angle $\phi_{0}(t)$ is given by

$$
\phi_{0}(t)=2 q_{t}(0),
$$

and is independent of the order $\left(n_{1}, n_{2}\right)$.

The modelling potential of the Ansatz (59) for the dynamics of tumour profiles lies in the fact that the cosine behaviour at large scales can be modelled independently of the deviations at the small scales. In particular, a suitable choice of the bounding function $q_{t}(s)$ allows to model any monotonically decreasing overlap $V_{t}(\phi)$ and, consequently, any monotonically decreasing deviation $d_{n_{1}, n_{2}}(t) f_{t}(\Delta \phi)$.

The assumption of a Brownian sheet in (59) is motivated by the implied order dependence of the amplitudes $b$ and $d$ in (60) and (61), respectively, and the fact that tumour profiles show self-scaling of spatial correlators (Schmiegel (2005b))

$$
c_{n_{1}, n_{2}}(t, \Delta \phi)=\left(c_{m_{1}, m_{2}}(t, \Delta \phi)\right)^{k_{t}\left[m_{1}, m_{2} ; n_{1}, n_{2}\right]}
$$

with self-scaling exponents $k_{t}$ of the form

$$
k_{t}\left[m_{1}, m_{2} ; n_{1}, n_{2}\right]=\frac{n_{1} n_{2}}{m_{1} m_{2}} .
$$

The self-scaling property (64) holds for all angular distances $\Delta \phi$ implying

$$
\frac{d_{n_{1}, n_{2}}(t)}{d_{m_{1}, m_{2}}(t)}=\frac{b_{n_{1}, n_{2}}(t)}{b_{m_{1}, m_{2}}(t)}=\frac{n_{1}, n_{2}}{m_{1}, m_{2}}
$$

in accordance with (60) and (61).

Figure 12 shows a comparison of star-shaped brain tumour profiles with simulations of the model (59). For the estimation of the parameters used for the simulation we refer to Schmiegel (2005b). 
Remark 4. The modelling framework (59) has been defined for the normalized radius $r_{t}(\phi)$. However, it equally applies to the modelling of the nonnormalized radius $R_{t}(\phi)$. The definition of correlators is invariant under rescaling with the mean radius. Going from $r_{t}(\phi)$ to $R_{t}(\phi)$ is equivalent to replacing $h(t)$ with $h(t)-\log \left(\mathrm{E}\left\{R_{t}(\phi)\right\}\right) / f_{t}(0)$, keeping all other parameters of the model (59) unchanged.

\section{Concluding remarks}

The modelling of the turbulent energy dissipation (44) and the turbulent velocity fields (29) and (37) within the class of ambit processes, as outlined in Section 5, poses various important questions, in addition to the purely mathematical problems listed in Section 2.5.

Of major interest is the identification of the parameters of the model with physical observables. For the temporal model (37) the intermittency process $J$ is identified with the local energy dissipation and as such accessible to data analysis. For the spatio-temporal model (29), the energy dissipation is identified with a weighted integral of the intermittency process.

Specifying suitable observables for the statistical analysis of the intermittency process $J$ are of great importance. The recently developed asymptotic theory of realised quadratic variation and its extension to realised multipower variation, see Barndorff-Nielsen et al (2005) and Barndorff-Nielsen and Shephard (2005) and references given there, is of relevance here.

The collapse of the densities of velocity increments at time scales $s$ as functions of the parameter $\delta(s)$ of the associated approximations within the class of NIG distributions indicates that $\delta(s)$ incorporates most of the individual characteristics of each experimental situation. From this point of view, the determination of the dependence of the weight function $g$ and the intermittency field $J$ in (29) on the function $\delta(s)$ should allow to model the evolution of the densities of velocity increments across scales in more detail.

Furthermore, the identification of $\delta(s)$ within the modelling framework is a first step towards a separation of non-universal features of the model, i.e. those that reflect the specific experimental situation, from universal features of the model that are independent of experimental details.

\section{Appendix: Normal inverse Gaussian distribution}

The normal inverse Gaussian law, with parameters $\alpha, \beta, \mu$ and $\delta$, is the distribution on the real axis $\mathbf{R}$ having probability density function

$$
p(x ; \alpha, \beta, \mu, \delta)=a(\alpha, \beta, \mu, \delta) q\left(\frac{x-\mu}{\delta}\right)^{-1} K_{1}\left\{\delta \alpha q\left(\frac{x-\mu}{\delta}\right)\right\} e^{\beta x}
$$


where $q(x)=\sqrt{1+x^{2}}$ and

$$
a(\alpha, \beta, \mu, \delta)=\pi^{-1} \alpha \exp \left\{\delta \sqrt{\alpha^{2}-\beta^{2}}-\beta \mu\right\}
$$

and where $K_{1}$ is the modified Bessel function of the third kind and index 1 . The domain of variation of the parameters is given by $\mu \in \mathbf{R}, \delta \in \mathbf{R}_{+}$, and $0 \leq|\beta|<\alpha$. The distribution is denoted by $\operatorname{NIG}(\alpha, \beta, \mu, \delta)$.

If $X$ is a random variable with distribution $\operatorname{NIG}(\alpha, \beta, \mu, \delta)$ then the cumulant generating function of $X$, i.e. $\mathrm{K}(\theta ; \alpha, \beta, \mu, \delta)=\log \mathrm{E}\left\{e^{\theta X}\right\}$, has the simple explicit form

$$
\mathrm{K}(\theta ; \alpha, \beta, \mu, \delta)=\delta\left\{\sqrt{\alpha^{2}-\beta^{2}}-\sqrt{\alpha^{2}-(\beta+\theta)^{2}}\right\}+\mu \theta .
$$

We note that the NIG distribution (67) has semiheavy tails; specifically,

$$
p(x ; \alpha, \beta, \mu, \delta) \sim \text { const. }|x|^{-3 / 2} \exp (-\alpha|x|+\beta x), x \rightarrow \pm \infty .
$$

The normal inverse Gaussian law can be characterized in terms of subordinated Brownian motion. For that, let $B_{t}$ be a Brownian motion starting at the point $\mu$ and having constant drift $\beta$. Let $Z_{t}$ be the inverse Gaussian Lévy process, assumed independent of the process $B_{t}$. The inverse Gaussian Lévy process is defined as the Lévy process for which $Z_{1} \stackrel{\operatorname{law}}{=} Z$ and where the distribution of $Z$ is the inverse Gaussian law whose probability density function is given by

$$
(2 \pi)^{-1 / 2} \delta e^{\delta \gamma} x^{-3 / 2} \exp \left\{-\left(\delta^{2} x^{-1}+\gamma^{2} x\right) / 2\right\} .
$$

This distribution is denoted $\operatorname{IG}(\delta, \gamma)$. Then, the process

$$
X_{t}=B_{z_{t}}+\mu t
$$

is also a Lévy process, termed the normal inverse Gaussian Lévy process, whose distribution at time $t=1$ is $\operatorname{NIG}(\alpha, \beta, \mu, \delta)$ where $\alpha=\sqrt{\beta^{2}+\gamma^{2}}$.

NIG shape triangle For some purposes it is useful, instead of the classical skewness and kurtosis quantities, to work with the alternative asymmetry and steepness parameters $\chi$ and $\xi$ defined by

$$
\chi=\rho \xi
$$

and

$$
\xi=[1+\bar{\gamma}]^{-1 / 2}
$$

where $\bar{\gamma}=\delta \sqrt{\alpha^{2}-\beta^{2}}$. Like $\bar{c}_{3}$ and $\bar{c}_{4}$, these parameters are invariant under location-scale changes and the domain of variation for $(\chi, \xi)$ is the normal inverse Gaussian shape triangle

$$
\{(\chi, \xi):-1<\chi<1,0<\xi<1\} .
$$

The distributions with $\chi=0$ are symmetric, and the normal and Cauchy laws occur as limiting cases for $(\chi, \xi)$ near to $(0,0)$ and $(0,1)$, respectively. Figure 13 gives an impression of the shape of the NIG distributions for various values of $(\chi, \xi)$. 


\section{References}

1. Barndorff-Nielsen, O.E. (1998a): Probability and Statistics; selfdecomposability, finance and turbulence. In L. Accardi and C.C. Heyde (Eds.): Proceedings of the Conference "Probability towards 2000", held at Columbia University, New York, 2-6 October 1995. Berlin: Springer-Verlag. Pp. 47-57.

2. Barndorff-Nielsen, O.E. (1998b): Processes of normal inverse Gaussian type. Finance and Stochastics 2, 41-68.

3. Barndorff-Nielsen, O.E., Blæsild, P. and Schmiegel, J. (2004): A parsimonious and universal description of turbulent velocity increments. Eur. Phys. J. B 41, 345-363.

4. Barndorff-Nielsen, O.E., Graversen, S.E., Jacod, J., Podolskij, M. and Shephard, N. (2006): A central limit theorem for realised power and bipower variations of continuous semimartingales. In Y. Kabanov and R. Lipster (Eds.): From Stochastic Analysis to Mathematical Finance, Festschrift for Albert Shiryaev. Berlin: Springer-Verlag. Pp. 33-68.

5. Barndorff-Nielsen, O.E. and Schmiegel, J. (2004): Lévy based tempo-spatial modelling; with applications to turbulence. Uspekhi Mat. Nauk 159, 63-90.

6. Barndorff-Nielsen, O.E. and Schmiegel, J. (2005): A stochastic differential equation framework for the turbulent velocity field. Research Report 2005-4. Thiele Centre, University of Aarhus. (Submitted.)

7. Barndorff-Nielsen, O.E. and Shephard, N. (2001): Non-Gaussian OrnsteinUhlenbeck-based models and some of their uses in financial economics (with Discussion). J. R. Statist. Soc. B 63, 167-241.

8. Barndorff-Nielsen, O.E. and Shephard, N. (2005): Variation, jumps, market frictions and high frequency data in financial econometrics. Prepared for the invited symposium on Financial Econometrics, 9th World Congress of the Econometric Society, London, 20th August 2005. (Under publication.)

9. Barndorff-Nielsen, O.E. and Shephard, N. (2006): Continuous Time Approach to Financial Econometrics. Cambridge University Press. (To appear.)

10. Benzi, R., Ciliberto, S., Tripiccione, R., Baudet, C., Massaioli, F. and Succi, S. (1993): Extended self-similarity in turbulent flows. Phys. Rev. E 48, R29-R32.

11. Brú, A., Pastor, J.M., Fernaud, I., Brú, I., Melle, S. and Berenguer, C. (1998): Super rough dynamics on tumor growth. Phys. Rev. Lett. 81 4008-4011.

12. Cairoli, R. and Walsh, J.B. (1975): Stochastic integrals in the plane. Acta Math. 134, 111-183.

13. Casciola, C.M., Benzi, R., Gualtieri, P., Jacob, B. and Piva, R. (2001): Double scaling and intermittency in shear dominated flows. Phys. Rev. E 65, 015301(R).

14. Carr, P., Geman, H., Madan, D. and Yor, M. (2003): Stochastic volatility for Lévy processes. Mathematical Finance 13, 345-382.

15. Castaing, B., Gagne, Y. and Hopfinger, E.J. (1990): Velocity probability density functions of high Reynolds number turbulence. Physica D 46, 177-200.

16. Cleve, J. and Greiner, M. (2000): The markovian metamorphosis of a simple turbulent cascade model. Phys. Lett. A 273, 104-108.

17. Cleve, J., Greiner, M. and Sreenivasan, K.R. (2003): On the effects of surrogacy of energy dissipation in determining the intermittency exponent in fully developed turbulence. Europhys. Lett. 61, 756-761.

18. Cleve, J., Greiner, M., Pearson, B.R. and Sreenivasan, K.R. (2004): Intermittency exponent of the turbulent energy cascade. Phys. Rev. E 69, 066316. 
19. Cont, R. and Tankov, P. (2004): Financial Modelling With Jump Processes. London: Chapman \& Hall/CRC.

20. Corsi, F., Kretschmer, U., Mittnik, S. and Pigorsch, C. (2005): The volatility of volatility. Unpublished working paper.

21. Eberlein, E. and Prause, K. (2002): The Generalized Hyperbolic Model: Financial Derivatives and Risk Measures. In H. Geman, D. Madan, S. Pliska and T. Vorst (Eds.): Mathematical Finance - Bachelier Congress 2000. Berlin: Springer-Verlag. Pp. 245-267.

22. Elsner, J.W. and Elsner, W. (1996): On the measurement of turbulence energy dissipation. Meas. Sci. Technol. 7, 1334-1348.

23. Forsberg, L. (2002): On the Normal Inverse Gaussian Distribution in Modelling Volatility in the Financial Markets. Uppsala University Press.

24. Frisch, U. (1995): Turbulence. The legacy of A.N. Kolmogorov. Cambridge University Press.

25. Hosokawa, I., Van Atta, C.W. and Thoroddsen, S.T. (1994): Experimental study of the Kolmogorov refined similarity variable. Fluid Dyn. Res. 13, 329-333.

26. Jensen, E.B.V, Jonsdottir, K.Y., Schmiegel, J. and Barndorff-Nielsen, O.E. (2006): Spatio-temporal modelling - with a view to biological growth. To appear in Statistics of Spatio-Temporal Systems, Monographs on Statistics and Applied Probability, Chapman \& Hall/CRC.

27. Jouault, B., Lipa, P. and Greiner, M. (1999): Multiplier phenomenology in random multiplicative cascade processes. Phys. Rev. E 59, 2451-2454.

28. Jouault, B., Greiner, M. and Lipa, P. (2000): Fix-point multiplier distributions in discrete turbulent cascade models. Physica D 136, 125-144.

29. Khoshnevisan, D. (2002): Multiparameter Processes. An Introduction to Random Fields. Heidelberg: Springer.

30. Klein, R. and Giné, E. (1975): On quadratic variation of processes with Gaussian increments. Ann. Prob. 3, 716-721.

31. Kolmogorov, A.N. (1941): Dissipation of energy in locally isotropic turbulence. Dokl. Akad. Nauk. SSSR 32, 16-18.

32. Kolmogorov, A.N. (1962): A refinement of previous hypotheses concerning the local structure of turbulence in a viscous incompressible fluid at high Reynolds number, J. Fluid Mech 13, 82-85.

33. Lindberg, C. (2005): Portfolio Optimization and Statistics in Stochastic Volatility Markets. Ph. D. Thesis, Chalmers University of Technology and Göteborg University.

34. Meneveau, C. and Sreenivasan, K.R. (1991): The multifractal nature of turbulent energy dissipation. J. Fluid Mech 224, 429-484.

35. Obukhov, A.M. (1962): Some specific features of atmospheric turbulence. J. Fluid Mech. 13, 77-81.

36. Rajput, B. and Rosinski, J. (1989): Spectral representations of infinitely divisible processes. Probab. Th. Rel. Fields 82, 451-487.

37. Schmiegel, J. (2005a): Self-scaling of turbulent energy dissipation correlators. Phys. Lett. A 337, 342-353.

38. Schmiegel, J. (2005b): Self-scaling tumor growth. Research Report 2005-7. Thiele Centre, University of Aarhus. (To appear in Physica A.)

39. Schmiegel, J., Cleve, J., Eggers, H.C., Pearson, B.R. and Greiner, M. (2004): Stochastic energy-cascade model for $(1+1)$-dimensional fully developed turbulence. Phys. Lett. A 320, 247-253. 
40. Schmiegel, J., Barndorff-Nielsen, O.E. and Eggers, H.C. (2006): A class of spatiotemporal and causal stochastic processes, with application to multiscaling and multifractality. South African Journal of Science 101, 513-519.

41. Shiryaev, A. N. (1999): Essentials of Stochastic Finance. Singapore: World Scientific.

42. Sreenivasan, K.R. and Antonia, R.A. (1997): The phenomenology of small-scale turbulence. Ann. Rev. Fluid Mech 29, 435-472.

43. Stolovitzky, G., Kailasnath, P. and Sreenivasan, K.R. (1992): Kolmogorov's refined similarity hypothesis. Phys. Rev. Lett. 69, 1178-1181.

44. Stolovitzky, G. and Sreenivasan, K.R. (1994): Kolmogorov's refined similarity hypotheses for turbulence and general stochastic processes. Rev. Mod. Phys. 66, 229-239.

45. Taylor, G.I. (1938): The spectrum of turbulence. Proc. R. Soc. Lond. A 164, 476-490.

46. Vincent, A. and Meneguzzi, M. (1991): The spatial structure and statistical properties of homogeneous turbulence. J. Fluid Mech. 225, 1-25.

47. Walsh, J.B. (1986a): An introduction to stochastic partial differential equations. In École d'Été de Probabilités de Saint-Flour XIV-1984. Berlin: Springer. Pp. 265-439.

48. Walsh, J.B. (1986b): Martingales with a multidimensional parameter and stochastic integrals in the plane. In Lectures in Probability and Statistics (Santiago de Chile 1986). Berlin: Springer. Pp. 329-491.

49. Wong, E. and Zakai, M. (1974): Martingales and stochastic integrals for processes with a multi-dimensional parameter. Z. Wahrscheinlichkeitstheorie verw. Geb. 29, 109-122.

50. Zhu, Y., Antonia, R.A. and Hosokawa, I. (1995): Refined similarity hypotheses for turbulent velocity and temperature fields. Phys. Fluids 7, 1637-1648.

51. Øigård, T.A., Hanssen, A., Hansen, R.E. and Godtliebsen, F. (2005): EMestimation and modelling of heavy-tailed processes with the multivariate normal inverse Gaussian distribution. Signal Processing 85, 1655-1673. 


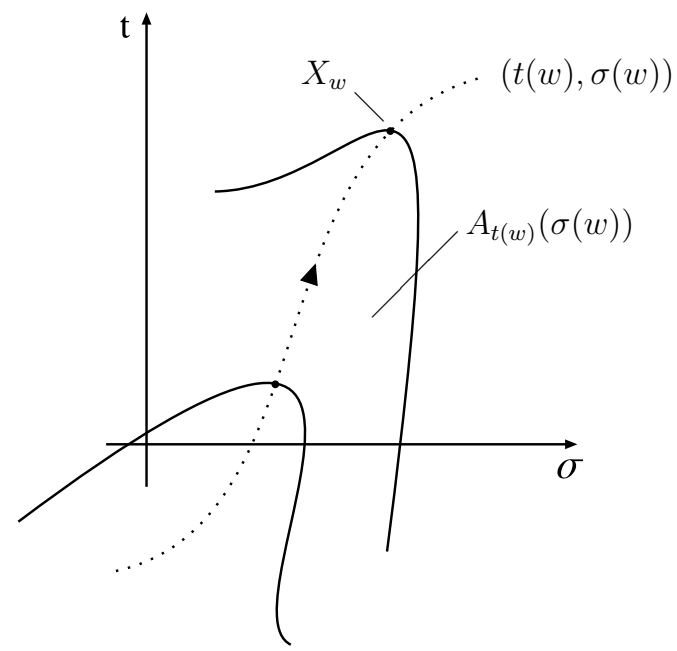

Fig. 1. Illustration of the dynamics of the process $X_{w}$ (15) along the curve $(t(w), \sigma(w))$.

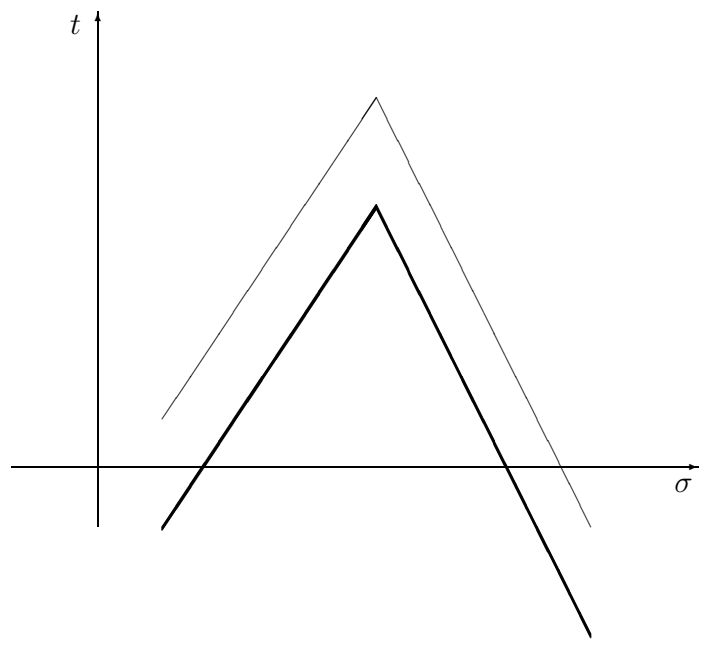

Fig. 2. Illustration of the dynamics of the process $X_{w}(15)$ along the curve $t(w)=w$, $\sigma(w)=\sigma$ constant. 


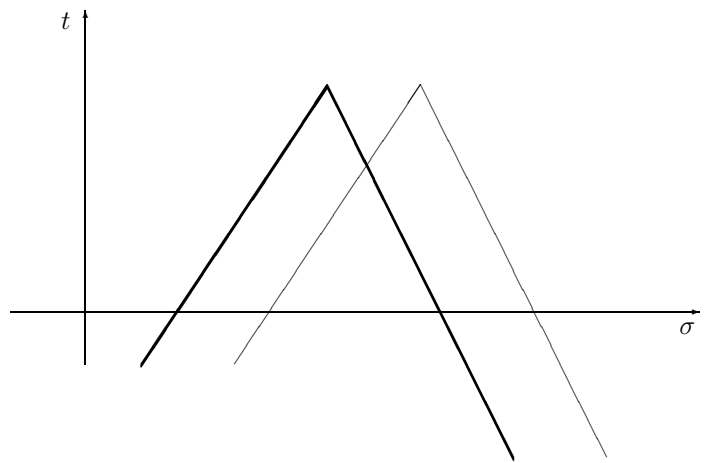

Fig. 3. Illustration of the dynamics of the process $X_{w}(15)$ along the curve $t(w)=t$ constant, $\sigma(w)=w$.

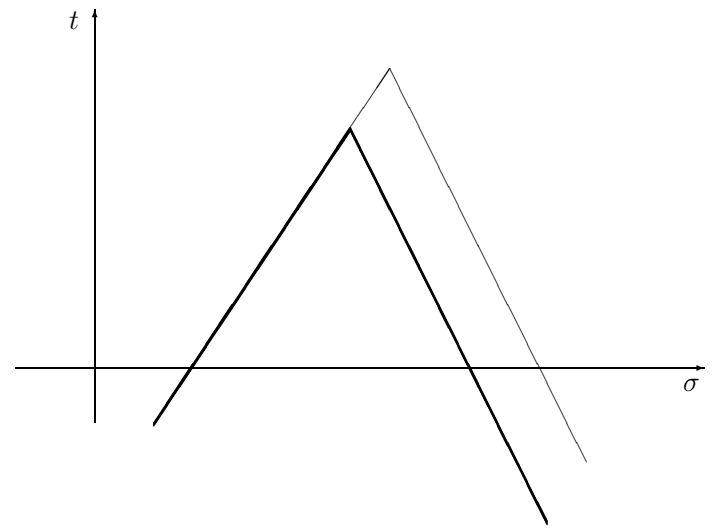

Fig. 4. Illustration of the dynamics of the process $X_{w}$ (15) along the curve $(t(w), \sigma(w))=\left(w, \sigma+c^{-} w\right)$. 
Ole E. Barndorff-Nielsen and Jürgen Schmiegel
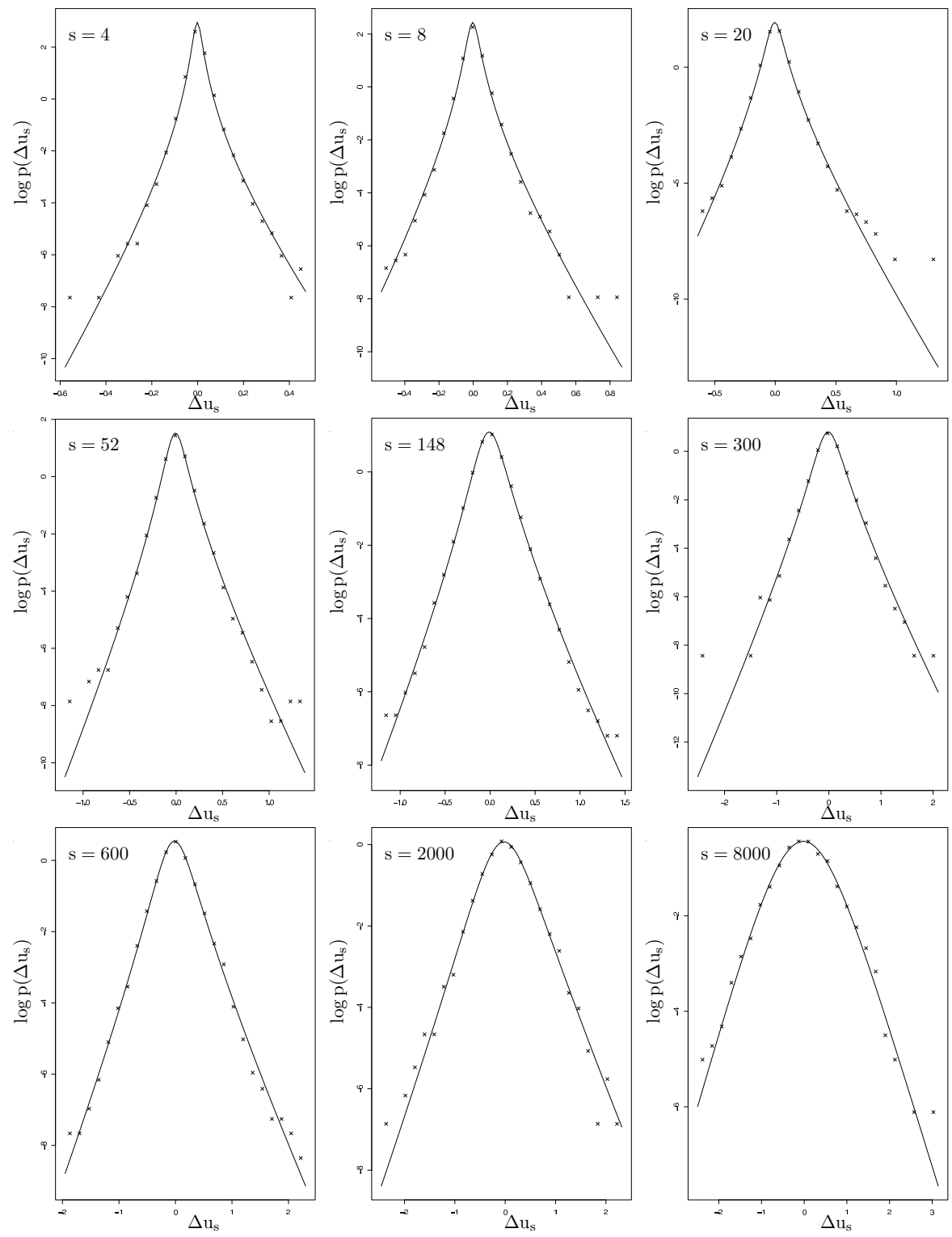

Fig. 5. Approximation of the pdf of velocity increments within the class of NIG distributions (solid lines, fitting by maximum likelihood) for data from the atmospheric boundary layer (kindly provided by K.R. Sreenivasan) with $R_{\lambda}=17000$ and time scales $s=4,8,20,52,148,300,600,2000,8000$ (in units of the finest resolution). 

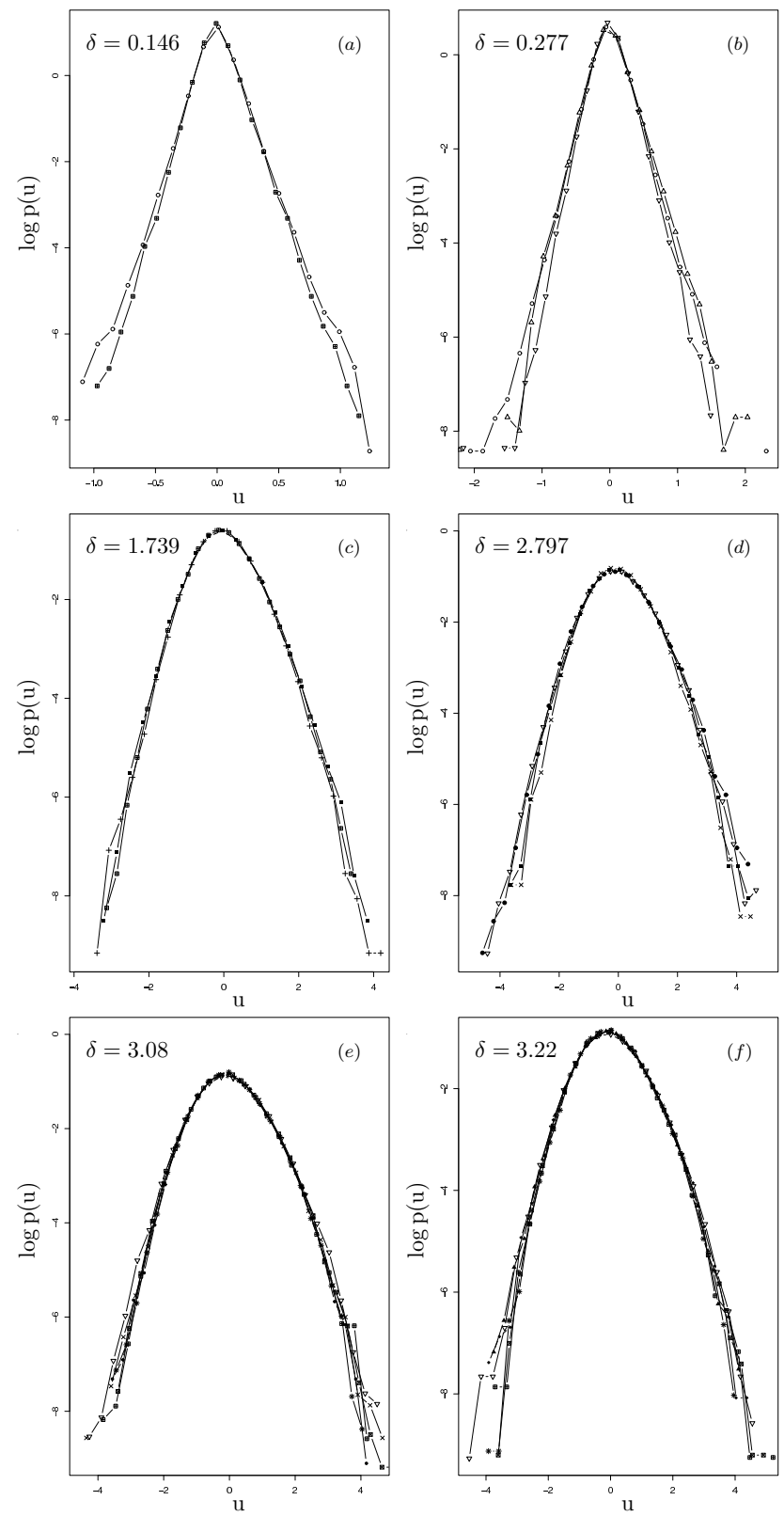

Fig. 6. Collapse of the densities of velocity increments at time scale $s$ for various fixed values of the scale parameter $\delta(s)$ of the approximating NIG-distributions. The data are from the atmospheric boundary layer (data set (at) with $R_{\lambda}=17000$, kindly provided by K.R. Sreenivasan), from a free jet experiment (data set (j) with $R_{\lambda}=190$, kindly provided by J. Peinke), from a wind tunnel experiment (data set (w) with $R_{\lambda}=80$, kindly provided by B.R. Pearson) and from a gaseous helium jet flow (data sets (h85), (h124), (h208), (h283), (h352), (h703), (h885), (h929), (h985) and (h1181) with $R_{\lambda}=85,124,208,283,352,703,885,929,985,1181$, respectively, kindly provided by $\mathrm{B}$. Chabaud). The corresponding values of the time scales $s$ (in units of the finest resolution of the corresponding data set) and the codes for the data sets are $(a)(s=116,($ at $))(\circ),(s=4,(\mathrm{~h} 352))(\boxplus),(b)(s=440,($ at $))(\mathrm{o})$, $(s=8,(\mathrm{j}))(\triangle),(s=8,(\mathrm{~h} 929))(\nabla),(c)(s=192,(\mathrm{~h} 885))(\mathbf{\square}),(s=88,(\mathrm{~h} 352))(\boxplus)$, $(s=10,(\mathrm{w}))(+),(d)(s=380,(\mathrm{~h} 885))(\boldsymbol{\square}),(s=410,(\mathrm{~h} 929))(\nabla),(s=350,(\mathrm{~h} 703))$ $(\times),(s=340,(\mathrm{~h} 985))(\bullet),(e)(s=420,(\mathrm{~h} 703))(\times),(s=440,(\mathrm{~h} 929))(\nabla),(s=$ $180,(\mathrm{~h} 352))(\boxplus),(s=270,(\mathrm{~h} 283))(\bullet),(s=108,(\mathrm{~h} 124))(*),(s=56,(\mathrm{~h} 85))(\otimes),(f)$ $(s=470,(\mathrm{~h} 929))(\nabla),(s=116,(\mathrm{~h} 124))(*),(s=60,(\mathrm{~h} 85))(\mathbb{\nabla}),(s=188,(\mathrm{~h} 352))$ $(\boxplus),(s=470,(\mathrm{~h} 1181))(\boldsymbol{\Lambda}),(s=140,(\mathrm{~h} 208))(\diamond)$. 


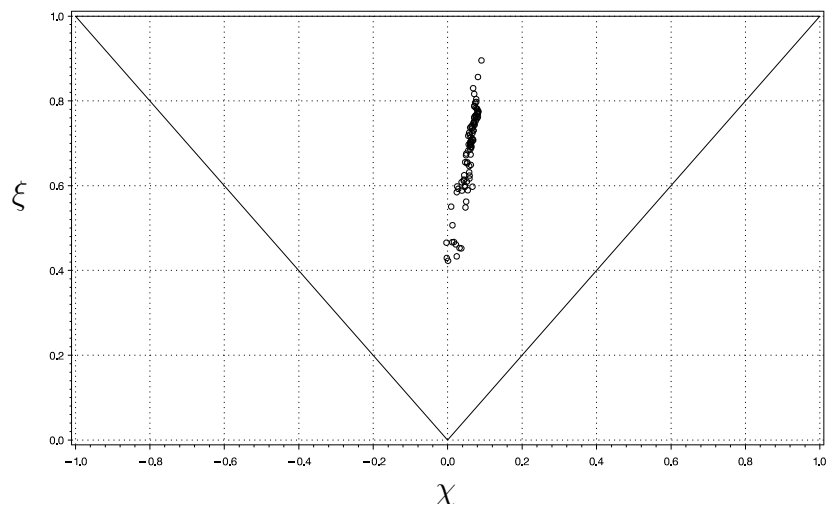

Fig. 7. Shape triangle for the evolution of the pdf of velocity increments across time scales (time scales increase from top to bottom) for data from the atmospheric boundary layer (kindly provided by K.R. Sreenivasan). 
Ambit processes; with applications to turbulence and tumour growth
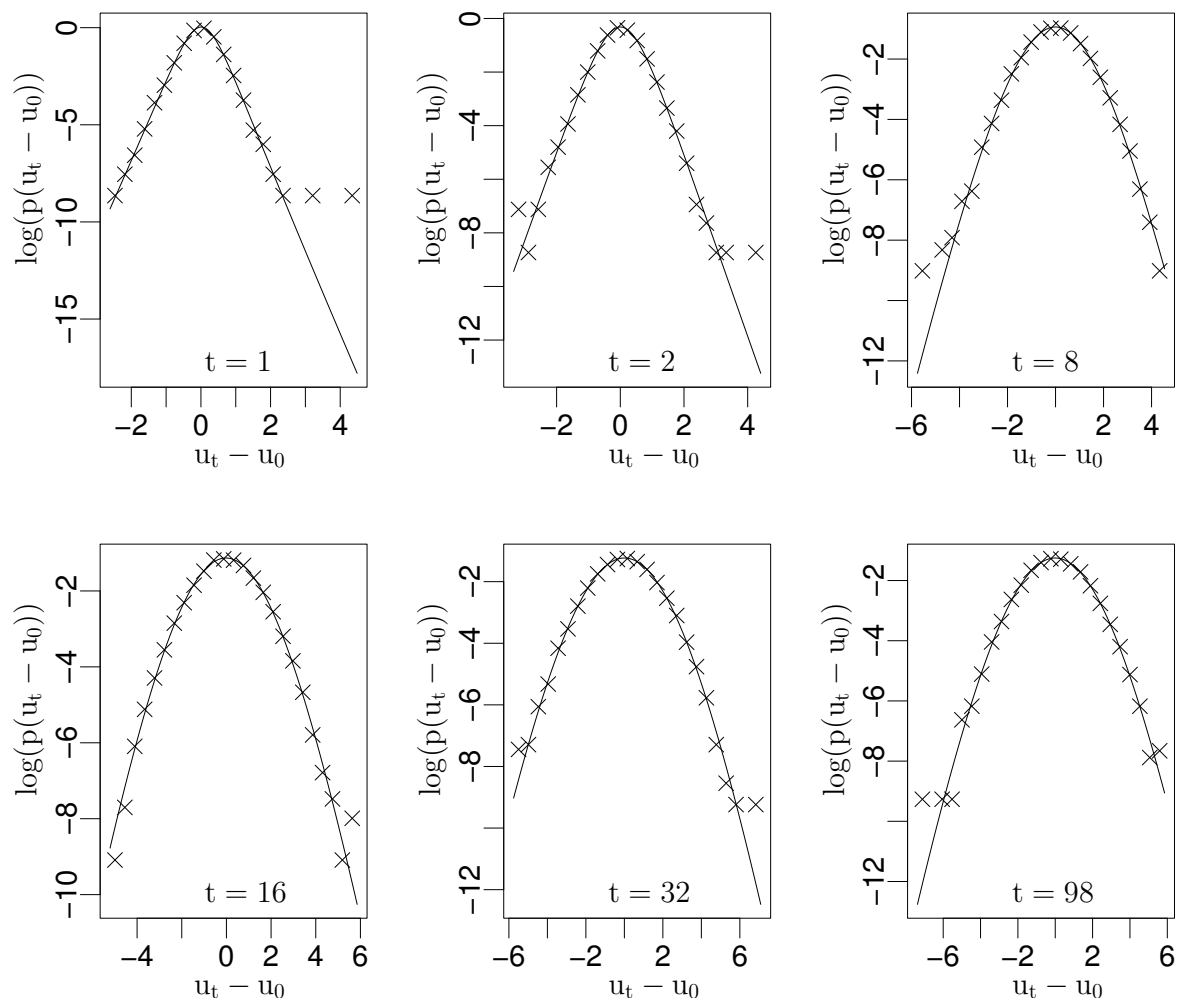

Fig. 8. Logarithm of the probability densities of the simulated increments $u_{t}-u_{0}$ (arbitrary units) under the model (37) with $t=1,2,8,16,32,98$ (in units of the finest resolution). The solid lines denote the approximation within the class of NIG distributions (fitting by maximum likelihood). 


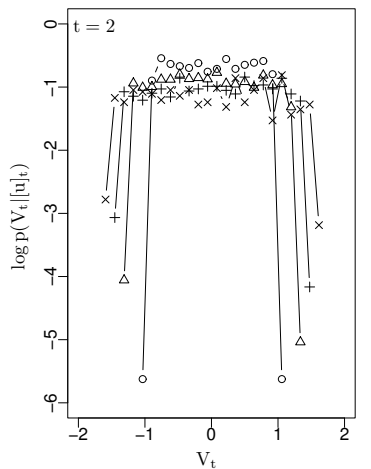

Fig. 9. Logarithm of the conditional densities $p\left(V_{t} \mid[u]_{t}\right)$ of the simulated Kolmogorov variable $V_{t}$ under the model (37) for $t=2$ (in units of the finest resolution) with $[u]_{t}^{1 / 3}=0.45(\circ),[u]_{t}^{1 / 3}=0.77(\triangle),[u]_{t}^{1 / 3}=0.99(+)$ and $[u]_{t}^{1 / 3}=1.20(\times)$ (in arbitrary units).

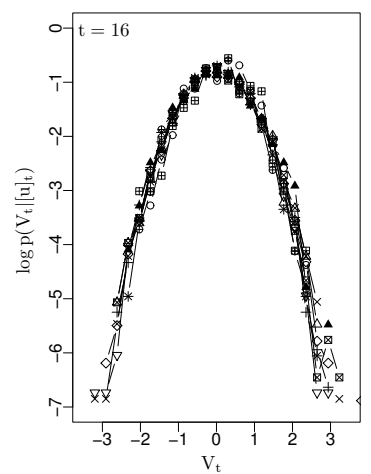

Fig. 10. Logarithm of the conditional density $p\left(V_{t} \mid[u]_{t}\right)$ of the simulated Kolmogorov variable $V_{t}$ under the model (37) for $t=16$ (in units of the finest resolution) with $[u]_{t}^{1 / 3}=0.98(\circ),[u]_{t}^{1 / 3}=1.16(\triangle),[u]_{t}^{1 / 3}=1.26(+),[u]_{t}^{1 / 3}=1.35(\times),[u]_{t}^{1 / 3}=1.44$ $(\diamond),[u]_{t}^{1 / 3}=1.53(\nabla),[u]_{t}^{1 / 3}=1.63(\otimes),[u]_{t}^{1 / 3}=1.72(*),[u]_{t}^{1 / 3}=1.81(\mathbf{\Delta})$, $[u]_{t}^{1 / 3}=1.9(\oplus)$ and $[u]_{t}^{1 / 3}=2.0(\boxplus)$ (in arbitrary units). 


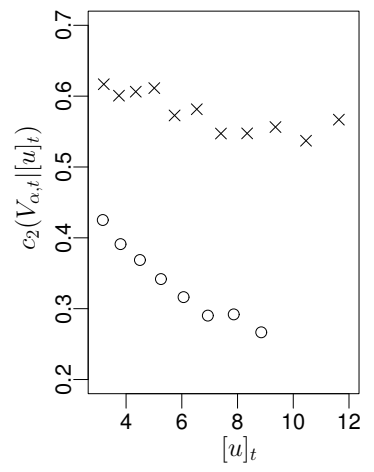

Fig. 11. Comparison of the simulated conditional variances $c_{2}\left(V_{\alpha, t} \mid[u]_{t}\right)$ for $\alpha=1 / 3$ $(\times)$ and $\alpha=1 / 2(\circ)$ as a function of $[u]_{t}$ (in arbitrary units) with $t=32$ (in units of the finest resolution).
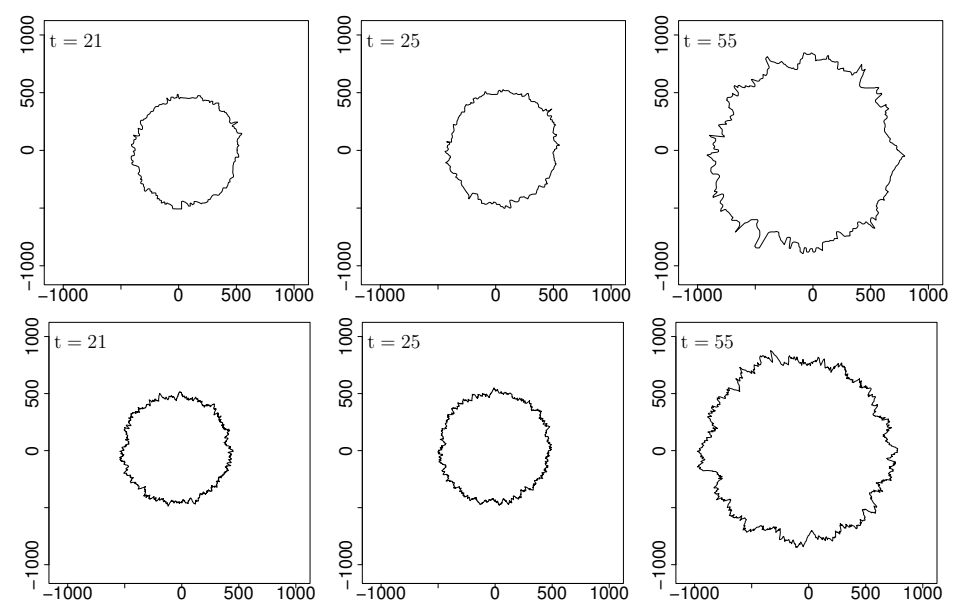

Fig. 12. Comparison of the simulated tumour profiles (bottom row) under the model (59) with the star-shaped tumour profiles (top row) at times $t=21,25,55$ (arbitrary units). 


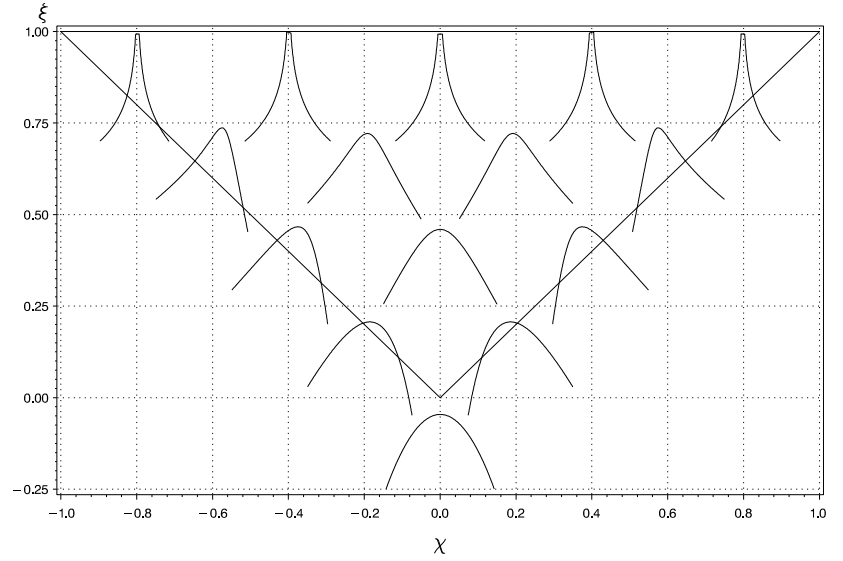

Fig. 13. The shape triangle of the NIG distributions with the log density functions of the standardized distributions, i.e. with mean 0 and variance 1 , corresponding to the values $(\chi, \xi)=( \pm 0.8,0.999),( \pm 0.4,0.999),(0.0,0.999),( \pm 0.6,0.75),( \pm 0.2,0.75)$, $( \pm 0.4,0.5),(0.0,0.5),( \pm 0.2,0.25)$ and $(0.0,0.0)$. The graphs of the log densities are placed at the corresponding values of $(\chi, \xi)$. 